\title{
Tropical Cyclone Evolution via Potential Vorticity Anomalies in a Three-Dimensional Balance Model
}

\author{
J. Dominique Möller and Michael T. Montgomery* \\ Meteorological Institute, Ludwig Maximilians University, Munich, Germany
}

(Manuscript received 15 June 1999, in final form 8 November 1999)

\begin{abstract}
A new mechanism of vortex intensification by convectively forced vortex Rossby waves was proposed by Montgomery and Kallenbach. As demonstrated by them, the axisymmetrization process is described by vortex Rossby waves that eventually propagate outward before their symmetrization. Montgomery and Kallenbach were able to relate these waves to intensity changes in barotropic hurricane-like vortices. In the present work these ideas are applied to better understand structure change and intensification of hurricanes in a baroclinic setting. The work of Möller and Montgomery, who examined the wave kinematics and wave-mean flow interaction of vortex Rossby waves in a barotropic model, is extended here to three dimensions. The model is based on the asymmetric balance theory of Shapiro and Montgomery. A nonlinear prognostic model is used to examine the effect of convectively generated potential vorticity (PV) disturbances on the evolution of a hurricane-like vortex on an $f$ plane. This investigation generalizes that of Montgomery and Enagonio, who studied tropical cyclogenesis using a quasigeostrophic balance model, to a larger Rossby number. Convection is represented to the extent that the prescribed initial PV anomalies could be convectively forced. As in this formulation gravity waves are excluded, the dynamics of vortex Rossby waves and their interaction with the mean vortex and each other can be focused upon.

Simple relaxation ("axisymmetrization") experiments with monochromatic azimuthal-wavenumber disturbances show that vortex Rossby waves propagate both radially and vertically. The higher the wavenumber the weaker the vertical propagation of the PV asymmetries and corresponding response of the basic state. Experiments where double-cluster PV anomalies are superimposed complement the cyclogenesis results of Montgomery and Enagonio. The lower-level cyclonic PV anomaly intensifies the vortex while symmetrizing for a wide range of anomaly amplitudes. Depending on the strength of the cluster, however, the upper-level anticyclonic PV anomaly is expelled outward (stronger anomaly), as in Montgomery and Enagonio, or is symmetrized (weaker anomaly) similar to the lower-level positive PV anomaly. When the ongoing process of convection is simulated by adding double-cluster PV anomalies to the PV fields (so-called pulsing), the tropical storm intensifies to hurricane strength whose intensity depends on the location and extent of the anomaly. These results confirm that there exists an alternative means of tropical cyclone intensification to the symmetric mode.
\end{abstract}

\section{Introduction}

Satellite observations indicate that episodic bursts of cumulus convection near the center of the incipient tropical vortex typically accompany tropical cyclone genesis. Spiraling bands of convection observed in the radar reflectivity field accompany the convective flare-ups. These observations suggest that the process of tropical cyclone formation is not axisymmetric, but rather an asymmetric one. When a tropical cyclone intensifies to

\footnotetext{
* Current affiliation: Department of Atmospheric Science, Colorado State University, Fort Collins, Colorado.
}

Corresponding author address: Dr. J. Dominique Möller, Meteorological Institute, Ludwig Maximilians University, 80333 Munich, Germany.

E-mail: nique@meteo.physik.uni-muenchen.de full strength, it generally acquires a higher degree of circular symmetry than in the formation stage. Yet, asymmetries of atmospheric or oceanic origin near the tropical cyclone eyewall region or environment are often observed to accompany rapid intensification and weakening events (e.g., Black et al. 1986). While some of these features may be incidental to tropical cyclone intensity change, others (such as sea surface temperature and upper-level troughs) are thought to be crucial. No consensus has yet been reached as to whether "internal" or "external" dynamics is key to subsequent development. But there is general agreement that a more complete understanding of asymmetric dynamics is required in order to more reliably predict tropical cyclone formation and intensity change.

Montgomery and Kallenbach (1997, henceforth MK) proposed a mechanism for hurricane intensity and structure change via vortex axisymmetrization of convectively generated asymmetric vorticity near the radius of 


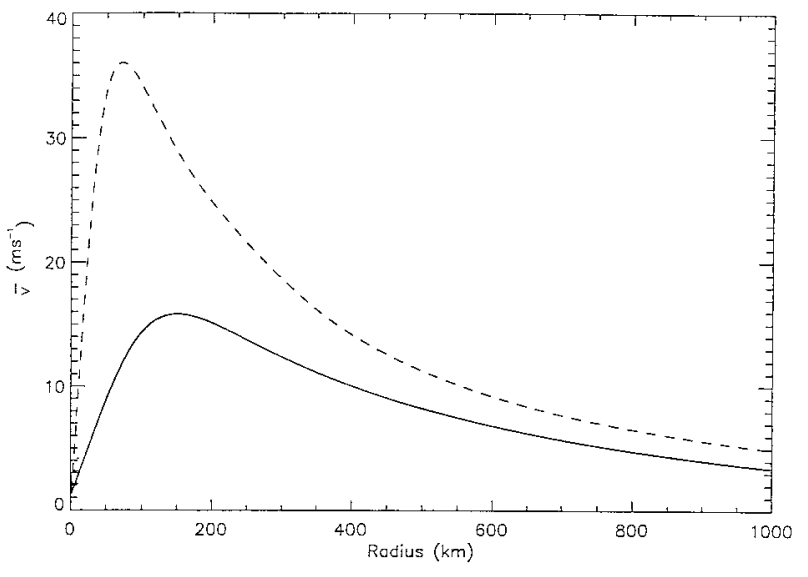

FIG. 1. Radial distribution of tangential velocity, $\bar{v}\left(\mathrm{~m} \mathrm{~s}^{-1}\right)$, for benchmark hurricane vortex (dashed line) and tropical storm (solid line); only the innermost $1000 \mathrm{~km}$ of the model domain is shown.

maximum tangential wind of the basic-state vortex. Although "axisymmetrization" has become known to be a universal process of vortex dynamics for monotonicmonopolar vortices (Melander et al. 1987; McCalpin 1987; Carr and Williams 1989; Sutyrin 1989; Guinn and Schubert 1993; Holland and Dietachmayer 1993; Ritchie and Holland 1993; Smith and Montgomery 1995), its significance in hurricane dynamics is now becoming more widely appreciated. As first demonstrated by MK, the axisymmetrization process is described by vortex Rossby waves that eventually propagate outward before their stagnation and symmetrization. Montgomery and Kallenbach (1997) were able to relate these waves to intensity changes in barotropic hurricane-like vortices. Our work extends the work of Möller and Montgomery (1999, henceforth MM), who examined the wave kinematics and wave-mean flow interaction of vortex Rossby waves in a barotropic shallow water balance model at large Rossby number. We apply these ideas to better understand structure change and intensification of hurricanes in three dimensions by imposing potential vorticity (PV) anomalies on a hurricane-like vortex. The investigation generalizes that of Montgomery and Enagonio (1998, henceforth ME), who studied tropical cyclogenesis using a quasigeostrophic balance model, to larger Rossby numbers. The present model is based on the asymmetric balance (AB) theory of Shapiro and Montgomery (1993, henceforth SM). As in SM, our experiments are performed on an $f$ plane, thereby explicitly excluding azimuthal wavenumber one contributions from the beta effect.

For some time now Pfeffer (1958; Challa and Pfeffer 1980; and several references in Challa et al. 1998) has suggested that while much of the tropical atmosphere during the hurricane season satifies the well-known necessary conditions for hurricane formation (Gray 1968), eddy processes in the storm environment are crucial for initiating hurricanes. The role of these eddies is to cause slight departures from gradient and hydrostatic balance on the large scale, which causes a secondary circulation that tries to reestablish balance. When eddy forcing acts favorably, an in-up-out transverse circulation results, supplying the requisite moisture to the core region of the incipient vortex. This theory has been shown to be able to discriminate between developing and nondeveloping disturbances (Challa et al. 1998) for several cases, yet the physical mechanism that actually spins up the vortex is not clear to us. Recent work of MK, ME, and MM elucidates a local eddy mechanism that can spin up an incipient vortex via convectively generated vortex Rossby waves. Here we will apply the vortex Rossby wave concept to examine the wave-mean flow intensity and structure change physics at tropical storm strength and higher. In the idealized approach of imposing PV anomalies, we avoid complications inherent in any moist convective parameterization. Convection is represented to the extent that the prescribed imposed initial PV anomalies could be convectively generated. We will show that vortex Rossby waves propagate not only radially but also vertically and will discuss the impact of the vertical propagation on structure and intensity change of hurricane-like vortices. Overall our results will confirm that there exists an alternative means of tropical storm intensification to the symmetric mode.

In section 2 we give an overview of the model used. Section 3 describes relaxation experiments with monochromatic wavenumbers. Section 4 discusses relaxation experiments with single- and double-cluster PV anomalies. Section 5 considers pulsing experiments (as in $\mathrm{ME}$ ) that are meant to mimic the process of episodic convection. Section 6 summarizes our findings.

\section{Asymmetric balance model}

The model is based on the asymmetric balance theory of SM. The nonlinear AB model was previously shown to give quantitatively similar predictions to the primitive equations (MM) in barotropic dynamics. Möller and Jones (1998) successfully applied the theory to a diagnosis of three-dimensional primitive equation model results. By contrast here we use a fully nonlinear threedimensional prognostic approach to examine the effect of convectively generated vorticity disturbances on the evolution of a hurricane-like vortex.

The geopotential tendency equation of the $\mathrm{AB}$ theory on an $f$ plane [cf. SM's Eq. (3.10)] is solved prognostically (see appendix). The linear evolution equation for the AB system is augmented to include weak nonlinearities, as proposed by SM (their section 5). The nonlinear terms [SM's Eq. (5.1)] are added to the right-hand side of the geopotential tendency equation. As shown in MM, a simple equation set for the asymmetric slow manifold within a rapidly rotating, inertially (centrifugally) stable, gravitationally subcritical (Froude number $<1$ ) vortex flow in two dimensions was obtained by evaluating the nonlinear terms $\left(\mathcal{N}_{r}\right.$ and $\mathcal{N}_{\lambda}$; see SM's section 5) in the 

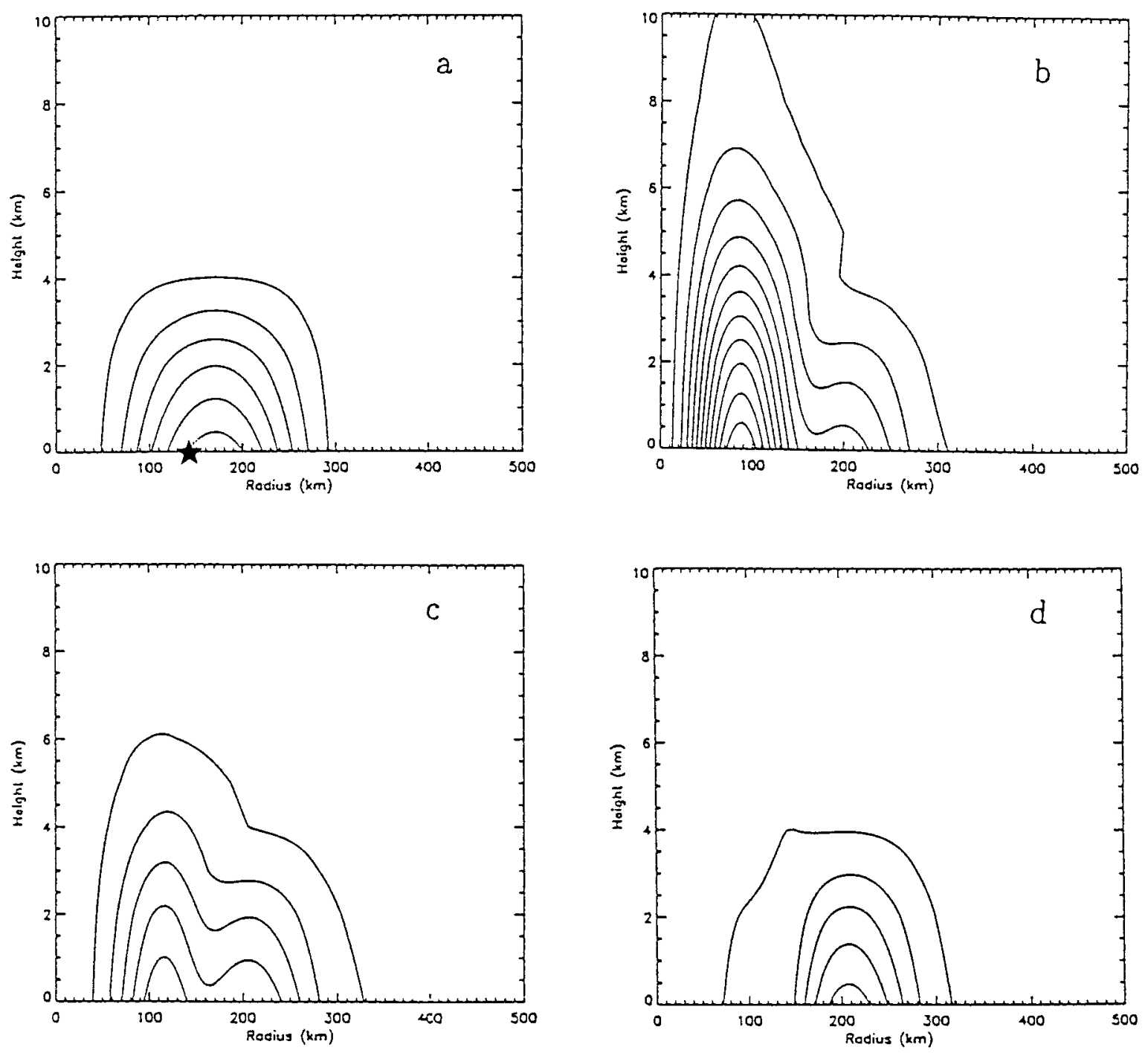

FIG. 2. Radius-height distribution of the PV asymmetry amplitude for the tropical storm vortex (a) initially for all wavenumbers, and after $8 \mathrm{~h}$ (b) for azimuthal wavenumber 1, (c) for azimuthal wavenumber 2, and (d) for azimuthal wavenumber 3. Contour interval $1 \times 10^{-9} \mathrm{~s}^{-3}$. Initial amplitude equals $60 \%$ of the PV at the RMW. The star in this and all succeeding figures indicates the location of the RMW.

radial and tangential equation with the pseudomomenta, $u_{\eta}^{\prime}=-[1 /(r \bar{\eta})] \partial \phi^{\prime} / \partial \lambda$ and $v_{\xi}^{\prime}=(1 / \bar{\xi}) \partial \phi^{\prime} / \partial r$ (see section 4 of SM) in place of the perturbation radial wind $u^{\prime}$ and tangential wind $v^{\prime}$, respectively, where $\phi^{\prime}$ denotes the perturbation geopotential, $\lambda$ the azimuth, $r$ the radius, $\bar{\eta}$ $=f+\bar{\zeta}$ the basic-state symmetric absolute vorticity, $f$ the Coriolis parameter, $\bar{\zeta}=r^{-1} d(r \bar{v}) / d r$ the basic-state relative vorticity, $\bar{v}$ the tangential wind, and $\bar{\xi}=f+$ $2 \bar{v} / r$, the inertia parameter.

Here we generalize the $\mathrm{MM}$ equation set to three dimensions by including the nonlinear term $\mathcal{N}_{\theta}$ (see SM's section 5) in the thermodynamic equation [Eq. (3.3) in SM] and the vertical advection terms in $\mathcal{N}_{r}$ and $\mathcal{N}_{\lambda}$ as well as $\mathcal{N}_{\theta}$. Note that for the evaluation of the nonlinear terms only the pseudomomenta and the linear version of $w^{\prime}$ [see SM's Eq. (3.9)] are used, whereas the full nonlinear forms (see appendix) are used when evaluating the perturbation velocities themselves. In all of our experiments $f=5 \times 10^{-5} \mathrm{~s}^{-1}$, except where noted, and the uniform background static stability $N^{2}$ $=1.2 \times 10^{-4} \mathrm{~s}^{-2}$. The vortex profile used for a hurricane-strength vortex is that of the benchmark vortex as described in MK (their Fig. 1), which is an initially cyclonic barotropic vortex in gradient balance with a maximum tangential wind $\bar{v}_{\max }=36.8 \mathrm{~m} \mathrm{~s}^{-1}$ at radius $r=70 \mathrm{~km}$ (Fig. 1). The profile used for a weaker tropical storm strength vortex is initially a cyclonic barotropic vortex in gradient balance with $\bar{v}_{\text {max }}=14.6 \mathrm{~m}$ $\mathrm{s}^{-1}$ at $145 \mathrm{~km}$ (Fig. 1). The vortex profiles are obtained by inversion of the basic-state PV of the nondimensional form $1+\alpha_{1} /\left(1+\alpha_{2} r^{2}+\alpha_{3} r^{3}\right)$, where $\alpha_{1}=60, \alpha_{2}=$ 0.2 , and $\alpha_{3}=1.0$ for the benchmark vortex, and $\alpha_{1}=$ $8, \alpha_{2}=0$, and $\alpha_{3}=0.1$ for the tropical storm [see Eq. (3.10) of Montgomery and Lu 1997]. The inversion of 

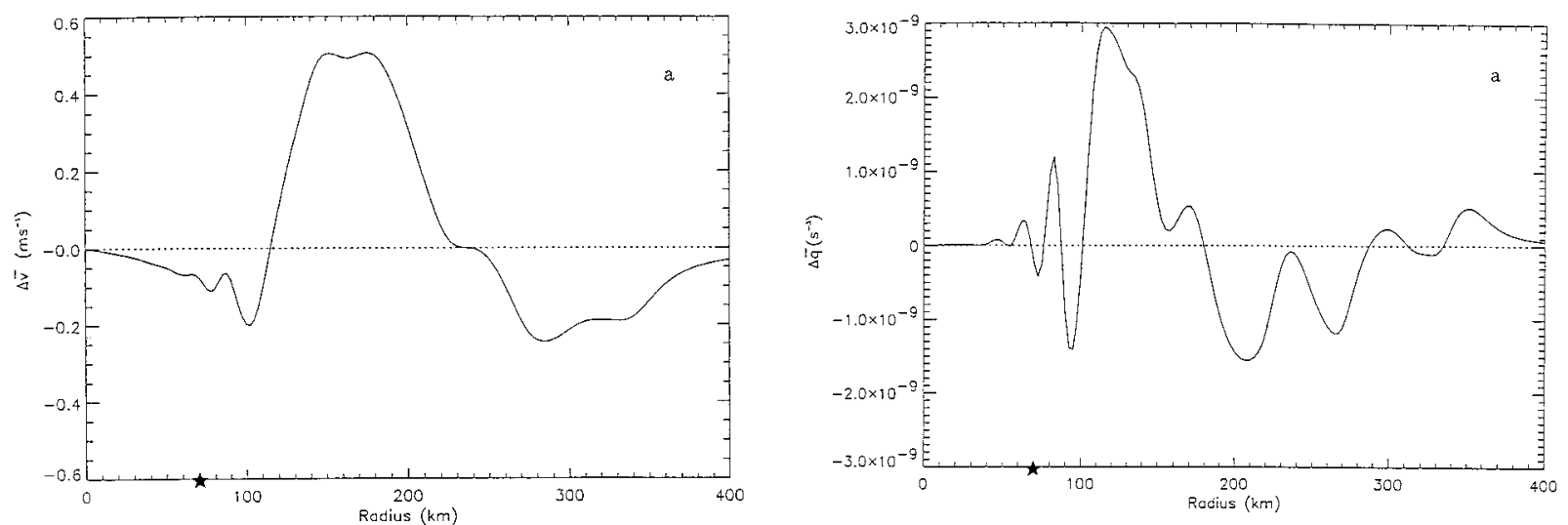

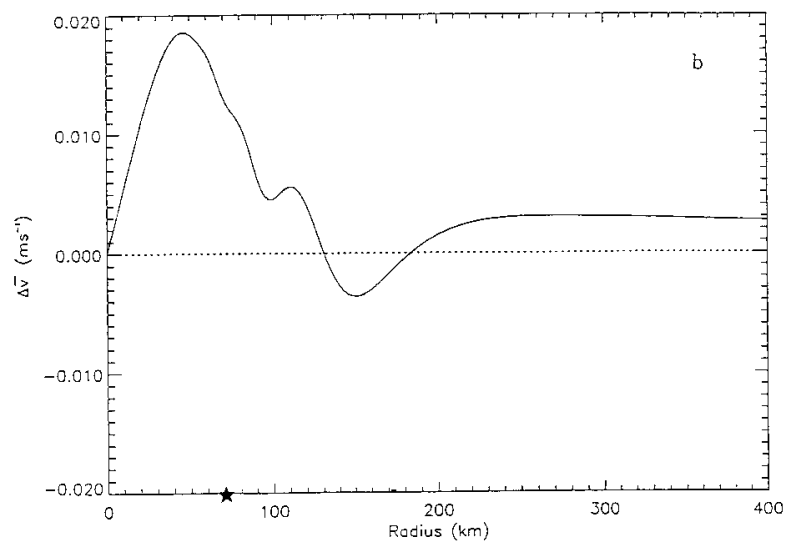

FIG. 3. Change of the basic-state tangential velocity, $\bar{v}\left(\mathrm{~m} \mathrm{~s}^{-1}\right)$, for the hurricane benchmark vortex after $6 \mathrm{~h}$ (a) at bottom of the domain $(z=0 \mathrm{~km})$, (b) at top of the domain $(z=10 \mathrm{~km})$, for initialization with azimuthal wavenumber 2 PV asymmetry, where amplitude equals $60 \%$ of the PV at the RMW.

the PV monopole is explained in detail in section 3c of Montgomery and Lu (1997). The vertical height of the domain is $H=10 \mathrm{~km}$ and the radial domain extends to $r=6000 \mathrm{~km}$ except for the experiments with monochromatic azimuthal wavenumber initialization (section 3 ), where a domain of $3000 \mathrm{~km}$ is used. The vertical grid spacing is $2 \mathrm{~km}$, and the radial grid spacing is 5 $\mathrm{km}$, except for the experiments in section 3 where the vertical grid spacing is $1 \mathrm{~km}$ and the radial $2.5 \mathrm{~km}$. Further numerical details are summarized in the appendix.

\section{Relaxation experiments with monochromatic PV anomalies}

We begin our study by examining the axisymmetrization process of initially monochromatic asymmetric disturbances on the tropical storm basic-state vortex of Fig. 1. The choice of monochromatic disturbances isolates the effect of each azimuthal wavenumber, which makes the experiment simpler and easier to understand.

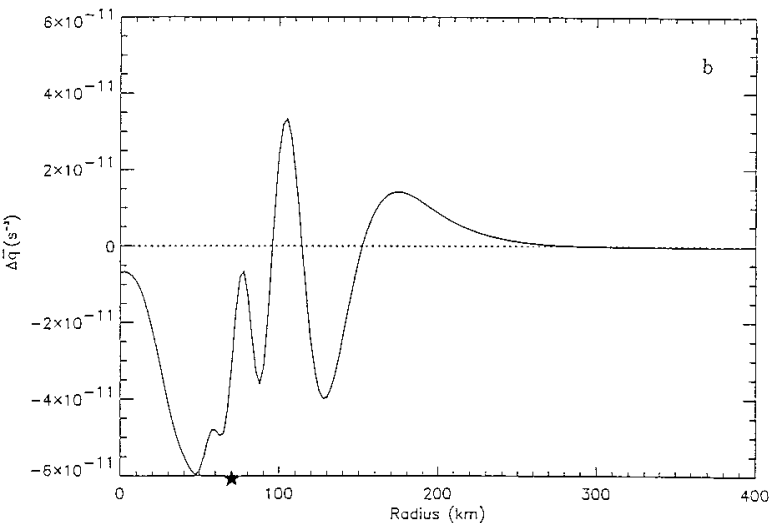

FIG. 4. As in Fig. 3 but change of the basic-state PV, $\bar{q}\left(\mathrm{~s}^{-3}\right)$, after $6 \mathrm{~h}$ (a) at bottom of the domain $(z=0 \mathrm{~km})$, (b) at top of the domain $(z=10 \mathrm{~km})$.

The PV disturbance is either an azimuthal wavenumber 1,2 , or 3 structure confined radially between $r=20$ and $320 \mathrm{~km}$, and levels below $5 \mathrm{~km}$. The PV disturbance is centered at a radius of $170 \mathrm{~km}$, with radial-height distribution given by $\alpha \sin ^{2}[\pi(r-20 \mathrm{~km}) /(300 \mathrm{~km})]$ $\cos (\pi z / H) \quad[z \leq 5 \mathrm{~km}]$, where $z$ is the pseudoheight (Hoskins and Bretherton 1972) and $\alpha$ the amplitude of the disturbance. The disturbance PV is given by the perturbation pseudo-PV

$q_{\Xi}^{\prime}=N^{2} \hat{\mathbf{k}} \cdot \boldsymbol{\nabla} \times \mathbf{u}_{\Xi}^{\prime}+\bar{\eta} \frac{\partial^{2} \phi^{\prime}}{\partial z^{2}}-\frac{\partial \bar{v}}{\partial z} \frac{\partial^{2} \phi^{\prime}}{\partial r \partial z}-\bar{\xi} \frac{\partial \bar{v}}{\partial z} \frac{\partial v_{\Xi}^{\prime}}{\partial z}$,

where the vertical component of pseudovorticity

$$
\zeta_{\Xi}^{\prime} \equiv \hat{\mathbf{k}} \cdot \nabla \times \mathbf{u}_{\Xi}^{\prime}
$$

is derived from the pseudomomenta based on the inertia parameter $\bar{\xi}$ :

$$
\mathbf{u}_{\Xi}^{\prime}=\left(u_{\Xi}^{\prime}, v_{\Xi}^{\prime}\right) \equiv\left(-\frac{1}{\bar{\xi}} \frac{1}{r} \frac{\partial \phi^{\prime}}{\partial \lambda}, \frac{1}{\bar{\xi}} \frac{\partial \phi^{\prime}}{\partial r}\right) .
$$

The initial pseudo-PV is inverted to obtain the perturbation geopotential $\phi^{\prime}$, where zero potential tempera- 

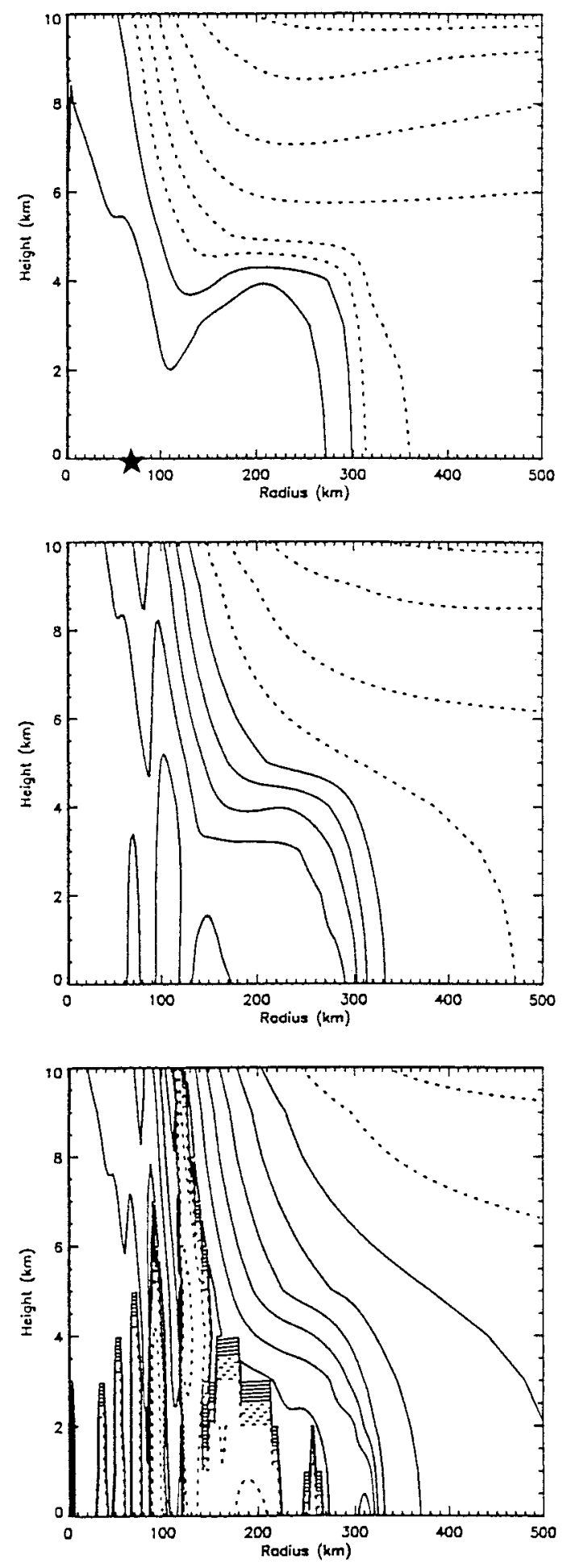

FIG. 5. As in Fig. 3 but radial-height plot of phase of azimuthal wavenumber $2 \mathrm{PV}$ anomaly after (a) 2, (b) 4, and (c) $6 \mathrm{~h}$. Contour interval is $15^{\circ}$; positive contours are solid and negative contours are dotted. The contours in the lower-left portion of (c) are noise due to the very small amplitude of the anomaly in that region. ture perturbations are used as boundary conditions at top and bottom (see appendix for more details). This choice of boundary conditions follows the classic decomposition of the flow into contributions from interior PV and from boundary potential temperature (Hoskins et al. 1985). While the specification of boundary conditions is not unambiguous and other choices are possible (e.g., Hakim et al. 1996), the use of zero surface potential temperature anomaly in the present study is based on observations of the tropical cyclone's thermal structure. The potential temperature in a tropical cyclone along the tropopause and sea surface is approximately constant compared to the interior potential temperature anomaly associated with the cyclone's warm core. Nearisothermal boundaries are found in Hawkins and Rubsam (1968) for Hurricane Hilda of 1964 (see discussion of Fig. 9 on their p. 623), in Palmèn and Newton (1969) for Hurricane Helene of 1958 (see their Fig. 15.6), as well as in Rotunno and Emanuel (1987) for a model hurricane (see their Figs. 5 and 8).

Montgomery and Kallenbach (1997) ME, and MM showed radial propagation of vortex Rossby waves and examined their impact on the azimuthal mean basic-state vortex. Similar to traditional Rossby waves (Charney and Drazin 1961) the propagation of vortex Rossby waves can be vertical as well. A local Wenzel-KramersBrillouin (WKB) analysis similar to that in MK (their section 2f) can be used to derive the dispersion relation for an unbounded baroclinic disturbance on a stably stratified barotropic vortex in gradient and hydrostatic balance. Analogous to Eq. (8) of MK, solutions of the form

$$
A(t) \exp [i(n \lambda+k(t)(r-R)+m(z-Z)-\Lambda(t))]
$$

are sought, where $A(t)$ is the time-dependent amplitude, $n$ is the azimuthal wavenumber, $m$ the vertical wavenumber, and $k$ the time-dependent radial wavenumber $\left[k=k_{0}-n t \bar{\Omega}^{\prime}(R)\right] ; \bar{\Omega}$ is the angular velocity of the basic-state vortex, $R$ the reference radius, $\bar{\Omega}^{\prime}$ the radial derivative of $\bar{\Omega}$ at $R, Z$ the reference height, and $\Lambda(t)$ the time-dependent phase. Then, for a barotropic basicstate vortex in a constant static stability atmosphere the local dispersion relation, which generalizes the dispersion relation of MK (see their section $3 \mathrm{~b}$ ) and includes the vertical structure of the disturbance, is given by

$$
\omega=n \bar{\Omega}+\frac{n}{R} \frac{\bar{\xi}}{\bar{q}} \frac{\bar{q}^{\prime}(R)}{\left[k^{2}+n^{2} / R^{2}+\left(\bar{\eta} \bar{\xi} m^{2}\right) / N^{2}\right]},
$$

where $\omega$ is the local wave frequency, and $\bar{q}^{\prime}$ the radial derivative of the basic-state PV at R. The corresponding vertical group velocity $\left(c_{g z}=\partial \omega / \partial m\right)$ is given by

$$
c_{g z}=-\frac{2 m n}{R} \frac{\bar{\eta}}{N^{2}} \frac{\bar{\xi}^{2}}{\bar{q}} \frac{\bar{q}^{\prime}(R)}{\left\{k^{2}+n^{2} / R^{2}+\left(\bar{\eta} \bar{\xi} m^{2}\right) / N^{2}\right\}^{2}} .
$$

For low-level PV forcing associated with convective heating we expect the waves to flux their energy upward $\left(c_{g z}>0\right)$. From (3.4) this implies that $m>0$ on cyclonic 

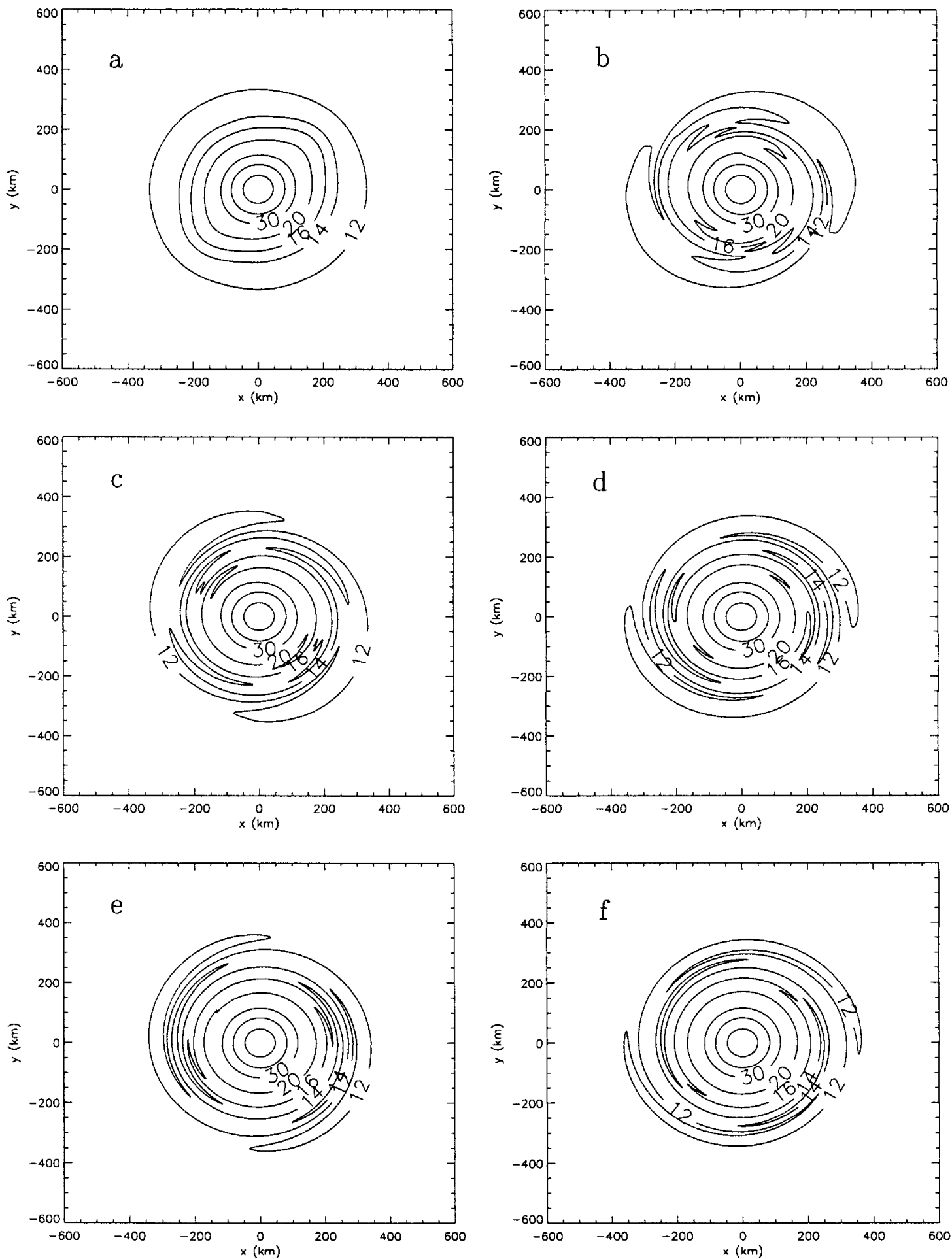

FIG. 6. Horizontal contour plot of total PV including double-cluster PV anomaly $\left(\times 10^{-9} \mathrm{~s}^{-3}\right)$ at the bottom of the domain $(z=0 \mathrm{~km})$ for the baroclinic tropical storm vortex (a) initially, (b) after 24, (c) 36, (d) 48, (e) 60, and (f) $72 \mathrm{~h}$. Initial amplitude equals $10 \%$ of $\bar{q}$ at the RMW. Positive contours are solid, and negative contours are dotted. 
monopoles, that is, the phase lines spiral cyclonically downward. Thus, upward propagating vortex Rossby waves on a cyclonic monopole tilt "westward with height" and produce a "northward heat transport" analogous to planetary Rossby waves. The stagnation height for a vertically propagating wave packet, derived analogously to the stagnation radius for radial propagation (MK's section 2f), is given by

$$
\begin{aligned}
z_{s}= & Z-\frac{m \bar{\eta} \bar{\xi}^{2} \bar{q}^{\prime}}{R N^{2} \bar{q}\left|\bar{\Omega}^{\prime}\right|} \frac{1}{B^{3}} \\
& \times\left[\frac{\pi}{2}+\frac{\bar{\Omega}^{\prime}}{\left|\bar{\Omega}^{\prime}\right|} \arctan \left(\frac{k_{0}}{B}\right)+\frac{\bar{\Omega}^{\prime}}{\left|\bar{\Omega}^{\prime}\right|} \frac{k_{0} / B}{1+k_{0}^{2} / B^{2}}\right],
\end{aligned}
$$

where

$$
B=\left(\frac{n^{2}}{R^{2}}+\frac{\bar{\eta} \bar{\xi} m^{2}}{N^{2}}\right)^{1 / 2} .
$$

For the initial-value experiments presented in this section the amplitude of the initial PV anomaly equals $60 \%$ of $\bar{q}$ at the radius of maximum wind (RMW). Experiments with linear and nonlinear versions of the model at such amplitudes result in basically the same vertical propagation characteristics for each azimuthal wavenumber, which confirms that the propagation of vortex Rossby waves, radially and vertically, on a PV monopole is essentially a linear process. The results presented here are from the nonlinear diagnostics so that the change of the basic-state quantities can be presented as well. Figure 2 shows vertical cross sections of the azimuthal wavenumber 1, 2, and 3 PV asymmetry amplitudes $\left(\left|\hat{q}_{\epsilon}(r, z, t)\right|\right.$, where hat denotes Fourier amplitude) initially and at $8 \mathrm{~h}$. Consistent with the dependence of $c_{g z}$ on the disturbance's spatial structure, for a disturbance not too radially or vertically confined (i.e., $k$ and $m$ not large) the asymmetries propagate farther upward to their vertical stagnation height, $z_{s}$, the lower the azimuthal wavenumber [Eq. (3.5)]. Inspection of Fig. 2 gives $z_{s} \sim 6 \mathrm{~km}$ for wavenumber 2 , and $z_{s} \sim 4 \mathrm{~km}$ for wavenumber 3 . For azimuthal wavenumber $1 z_{s} \sim 10$ $\mathrm{km}$, though the presence of a "pseudomode" near the vortex center due to the motion of the basic-state vortex (see MK and MM) may contribute.

Gall et al. (1998) note that "we have no information on the tangential momentum or angular momentum transport by the spirals [small-scale spirals observed in hurricanes], either horizontally or vertically." While observations may currently lack the requisite spatio-temporal resolution to thoroughly assess this issue the present work does provide some useful insight in this area. Since vortex Rossby waves propagate upward and transport energy upward as well, they can in principle change the upper-level tangential velocity by transporting momentum from the lower levels into the upper levels of the vortex by eddy fluxes. In order to examine the change of the basic-state vortex induced by the exci- tation and propagation of vortex Rossby waves we choose an experiment where the model is initialized with the barotropic benchmark hurricane vortex and a superimposed azimuthal wavenumber 2 PV asymmetry, as described above. Qualitatively the results are the same as with the tropical storm, but due to the stronger inertial terms for the benchmark vortex the upward energy transport tends to be stronger and easier to observe. ${ }^{1}$ We focus on the wavenumber 2 PV asymmetry as it results in a stronger response than higher wavenumbers due to the larger radial and vertical group velocities (see above) and it is easier to diagnose its wave propagation characteristics in the absence of the pseudomode that would be present with a wavenumber 1 disturbance. Figure $3 \mathrm{a}$ shows that after $6 \mathrm{~h}$ the azimuthally averaged tangential velocity, $\bar{v}$, decreases inside and increases and decreases again outside the RMW at lower levels as in the barotropic case (see MK and MM). By this time the anomaly is axisymmetrized and the change of the basic state has reached its maximum. This result can be explained by diagnostics of waveactivity propagation in the radial direction (Shapiro 2000). The weak decrease at the center is due to the excitation of interior vortex Rossby waves through the advection of basic-state PV by perturbation radial velocity (see MK and MM). At upper levels, however, $\Delta \bar{v}$ is much weaker and $\bar{v}$ increases most inside the RMW (Fig. 3b). These changes in $\bar{v}$ will contribute to an inward tilt of the vortex with height. The corresponding changes in $\bar{q}$ tend to increase the PV near $130-\mathrm{km}$ radius at lower levels (Fig. 4a) and deplete it near this radius and inside the RMW at upper levels (Fig. 4b). The vertical propagation of the PV disturbance is confirmed in Fig. 5, which shows the evolution of the phase $(\Lambda)$. The outward and upward decrease in phase is consistent with the radial wavenumber $k>0$ (trailing spirals) and vertical wavenumber $m>0$, which gives an inward tilt of the phase lines with height. As can be seen in this figure the PV disturbance propagates both radially outward and upward from the initial disturbance. In a stable vortex $\left(q^{\prime}<0\right)$ this propagation is consistent with both outward $\left(c_{g r}>0\right)$ and upward $\left(c_{g z}>0\right)$ energy propagation. Nevertheless horizontal eddy vorticity fluxes are present initially at every level. Thus, the dependence of $\Delta \bar{v}$ on $z$ may not only be due to vertical propagation but also may be partly a reflection of a vertically dependent horizontal eddy vorticity flux, due to the initial PV anomaly.

\section{Relaxation experiments with localized PV anomalies}

a. Relaxation on a midlevel vortex

In ME's quasigeostrophic experiments, localized PV anomalies, so-called single clusters or double clusters,

\footnotetext{
${ }^{1}$ For disturbances that are relatively deep, $c_{g z}$ [Eq. (3.4)], is approximately proportional to $\overline{\eta \xi}^{2}$ and so is larger the stronger the vortex.
} 
are superimposed near a larger-scale parent vortex. Following ME the vertical structure of the anomaly is given by $\alpha \cos (\pi z / H)$, although in the present work the amplitude $\alpha$ varies between $10 \%$ and $60 \%$ of the initial basic-state PV at the RMW. These amplitudes, which are chosen so as to remain in the quasi-linear regime, result in a weak asymmetric and symmetric response; larger amplitudes are used in section 5. The horizontal structure is that of a double cluster (Figs. 6a-9a), as described in sections 3.3 and 4.2 of $\mathrm{ME}$, and has the form

$$
e^{-\beta r_{1}^{2}}+e^{-\beta r_{2}^{2}}
$$

where $r_{1}^{2}=\left[\left(x-x_{c}\right)^{2}+\left(y-y_{c}\right)^{2}\right] / r_{\max }^{2}, r_{2}^{2}=[(x+$ $\left.\left.x_{c}\right)^{2}+\left(y+y_{c}\right)^{2}\right] / r_{\max }^{2}$, with $\left(x_{c}, y_{c}\right)$ the vortex center, and $r_{\max }$ the RMW. For the experiments in this subsection $\beta=2.5$, and $\left(x_{c}, y_{c}\right)=(0.88,0.88) r_{\max }$, which corresponds to anomaly centers at $r=180 \mathrm{~km}$ and a radial $e$-folding width ${ }^{2}$ of $185 \mathrm{~km}$. ME's upper-level negative PV anomaly was expelled outward whereas the lower-level positive PV anomaly was symmetrized for a wide range of PV anomaly amplitudes. The negative upper-level PV anomalies formed a tripole (Polvani and Carton 1990; Orlandi and van Heijst 1992; ME) whose dynamical origin could be partly explained by the existence of discrete neutral (i.e., marginally stable) or unstable vortex Rossby modes (MK, MM). In order to investigate what impact the strength of the PV disturbance has on our basic-state vortex we initialize, similar to $\mathrm{ME}$, with asymmetric PV anomalies (negative at upper levels, positive at lower levels) of different strength and examine the ensuing nonlinear dynamics. In contrast to ME we superimpose only a double-cluster PV anomaly which, unlike a single cluster, does not move the center of the vortex. ${ }^{3}$ The parent vortex is a tropical storm as described in section 2, and in order to compare our results with those of ME we take $f=10^{-4} \mathrm{~s}^{-1}$ in this section. ${ }^{4}$

The basic-state vortex employed in this subsection is that of a midlevel vortex, with the maximum tangential wind $\left(\bar{v}_{\max }=22.0 \mathrm{~m} \mathrm{~s}^{-1}\right)$ occurring at the middle level $[1+0.5 \sin (\pi z / H)] .^{5}$ As an example, Figs. 6-9 show contour plots of the total PV at successive times at the top and bottom of the domain for the different PV anomaly initializations for the midlevel basic state vortex.

For the PV anomaly of $10 \%$, Fig. 6 shows the axisymmetrization of the lower-level positive PV asym-

\footnotetext{
2 The $e$-folding width is the radial distance between the locations where the amplitude is $1 / e$ of the maximum.

${ }^{3}$ Relaxation experiments with a single-cluster anomaly gave qualitatively similar results, and for brevity of presentation only doublecluster anomalies are presented here.

${ }^{4}$ The experiment with an initially barotropic vortex and a PV amplitude $60 \%$ of the PV at the RMW and $f=5 \times 10^{-5} \mathrm{~s}^{-1}$ gave quantitatively the same result as the experiment with $f=10^{-4} \mathrm{~s}^{-1}$.

5 The results for an initially barotropic vortex (not shown) are very similar to the ones with an initially baroclinic vortex.
}

metries and Fig. 7 the upper-level negative PV asymmetries in horizontal cross sections. For both levels the radius at which the outermost vortex Rossby wave packet stagnates, the radial stagnation radius (for more details see $\mathrm{MK}$ and $\mathrm{MM}$ ) is at $\sim 300 \mathrm{~km}$. For the same experiment where the PV asymmetry amplitude is $60 \%$ of the PV at the RMW, the lower-level contour plot (Fig. 8) gives a very similar result to the experiment with $10 \%$ amplitude (Fig. 6). The upper-level contour plot (Fig. 9), however, is different from the weaker case (Fig. 7), as the PV asymmetries move radially outward and form a tripole (Figs. 9e,f), in part due to the change of sign of the basic-state radial PV gradient (not shown) as in ME.

Independent of the strength of the initial PV anomalies the lower-level positive PV anomalies are always symmetrized into the basic-state vortex (as in ME). On the other hand, depending on the strength of the cluster the upper-level anticyclonic PV anomaly is expelled outward (stronger anomaly) and forms a tripole, as in the cyclogenesis experiments of $\mathrm{ME}$, or is symmetrized (weaker anomaly) while it propagates outward similar to the lower-level positive PV anomaly. Montgomery and Enagonio (1998) made sensitivity tests concerning the magnitude and location of the asymmetries and found that in their experiments the upper-level PV disturbance was never axisymmetrized, in part due to the azimuthal mean radial wave-induced sign change of the PV gradient and the excitation of neutral (marginally stable) or unstable vortex Rossby modes. They decreased the amplitude of the superimposed PV anomaly "by a factor of five from the nominal case and still obtained a weak wave-induced sign change of the azimuthal radial PV gradient at upper levels." In the experiments here, by contrast, the amplitude of the PV disturbance at upper levels is decreased by a factor of six (to $10 \%$ of the PV at the RMW). Due to the weakness of the initial anomaly, the basic-state PV gradient does not change sign and no neutral or unstable mode is observed. Consequently, rather than forming a tripole, the smaller amplitude negative PV anomaly gets symmetrized.

The symmetrizing PV anomaly induces changes in the primary circulation analogous to those in the barotropic experiments (cf. footnote 5). Both experiments with the initially barotropic and baroclinic vortices exhibit very similar changes in the primary circulation, but only the ones for the baroclinic vortex will be presented. The acceleration of the circulation near the center of the anomaly can be readily explained by wave activity ideas (see ME and section $3 \mathrm{~b}$ of MM). Consistent with the outward propagation of the PV asymmetries the changes of the basic-state vortex move more or less outward in time depending on the strength of the PV anomaly. The changes of the tangential velocities $\Delta \bar{v}$ at upper and lower levels after $24 \mathrm{~h}$ are shown in Fig. 10a for the weaker anomaly (10\%). The maximum 

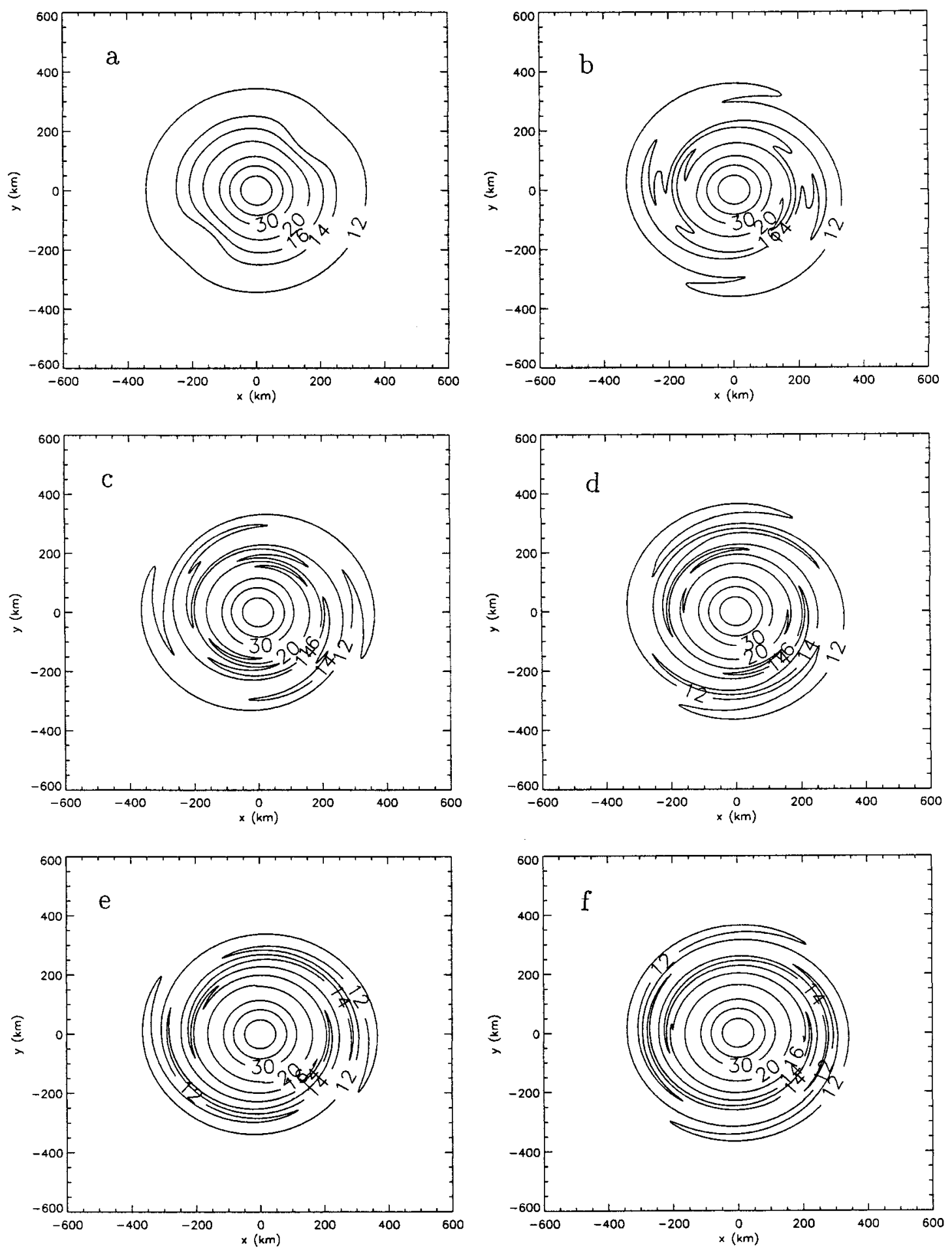

FIG. 7. As in Fig. 6 but at the top of the domain $(z=10 \mathrm{~km})$.

of $\Delta \bar{v}$ for the top (dashed line) and bottom (solid line) are displaced only $\sim 20 \mathrm{~km}$ from each other. For the stronger anomaly $(60 \%)$ after $24 \mathrm{~h}$ (Fig. 10b) the maximum of $\Delta \bar{v}$ is displaced $80 \mathrm{~km}$ outward at upper levels (dashed line) from the lower level (solid line) as the upper PV anomaly moves radially more outward than in the weak anomaly case (cf. Figs. $9 b$ and 7b). The strength of the PV anomalies in these relaxation experiments influences not only the strength of the basicstate change but also the location of the mean flow 

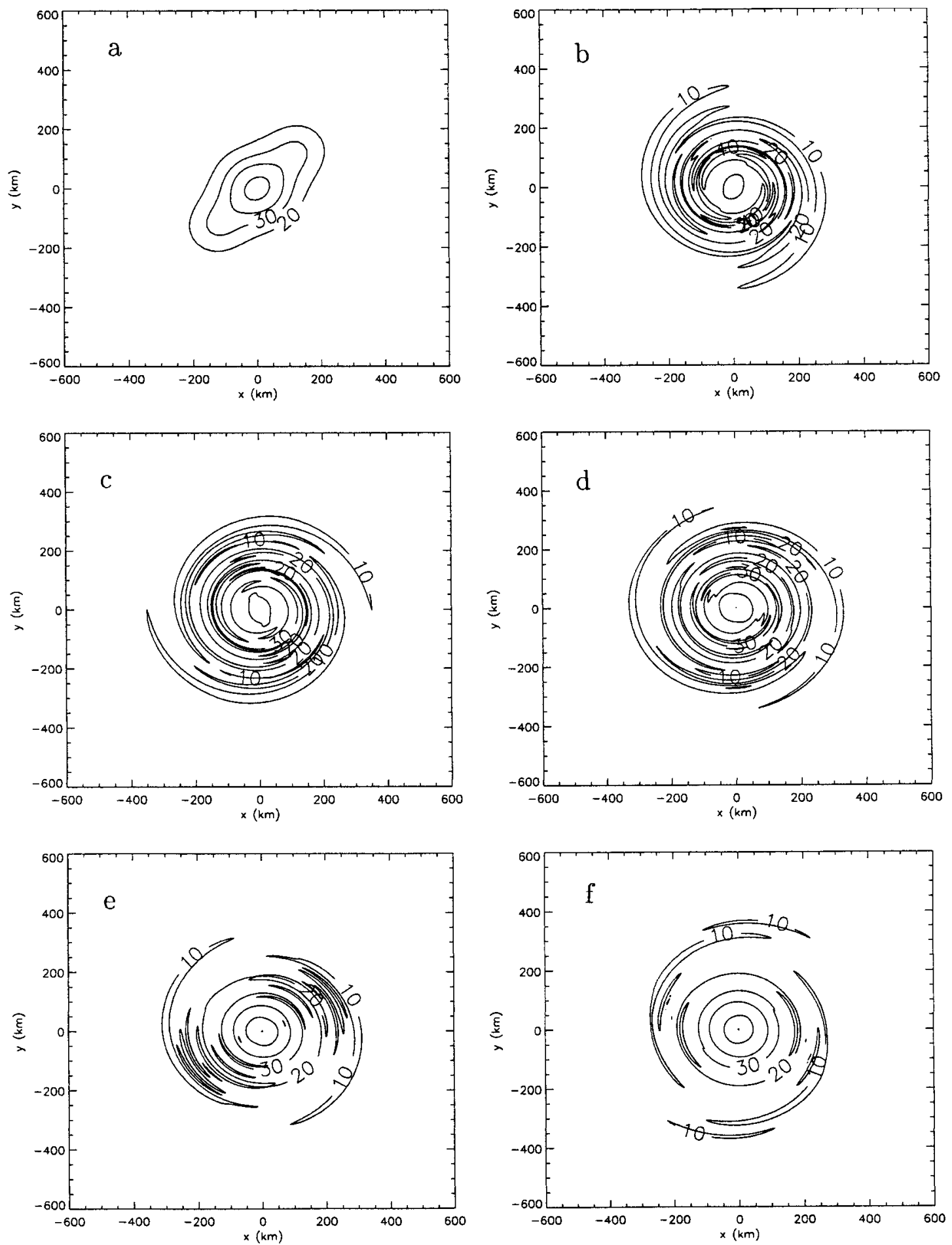

FIG. 8. As in Fig. 6 but initial amplitude equals $60 \%$ of $\bar{q}$ at the RMW.

response. The weaker the anomaly the more the basicstate change occurs where the PV disturbance is initially superimposed. When the PV disturbance is relatively strong the basic-state change occurs farther outside the location of the initial disturbance, which influences the structure and intensity of the basic-state vortex. This is in contrast to a wavenumber $1 \mathrm{PV}$ asymmetry initialization, as described in detail in ME, where the stronger the anomaly the closer the mean flow response is to the center of the vortex. 

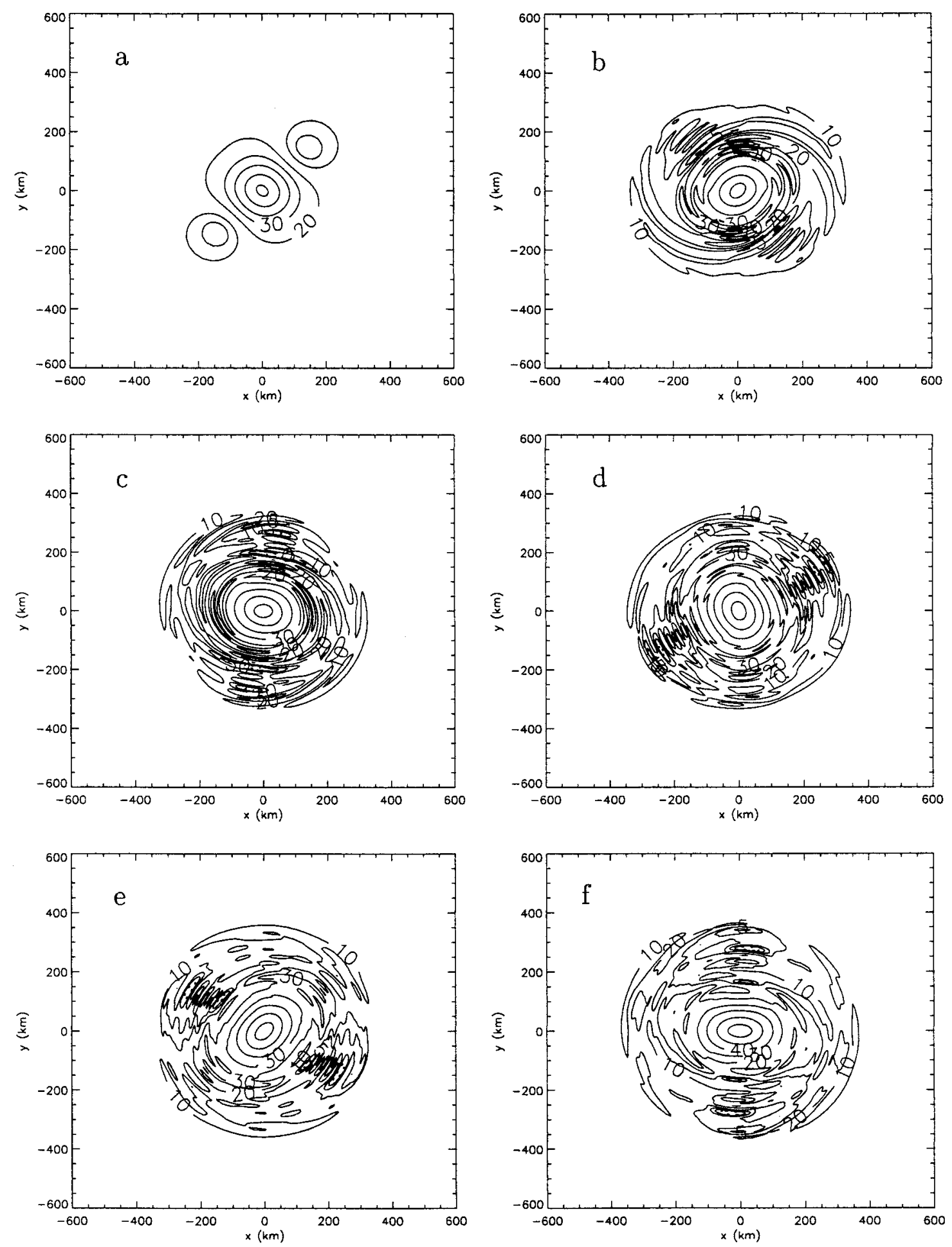

Fig. 9. As in Fig. 7 but initial amplitude equals $60 \%$ of $\bar{q}$ at the RMW.

\section{b. Induced secondary circulation}

In addition to the change of the mean primary circulation, the disturbance induces a mean secondary circulation that generally tends to counteract the changes in the primary one. In the relaxation experiments presented here the mean secondary circulation is strongest between 6 and $12 \mathrm{~h}$ and then decreases rapidly. As the baroclinic structure of the disturbance complicates the 

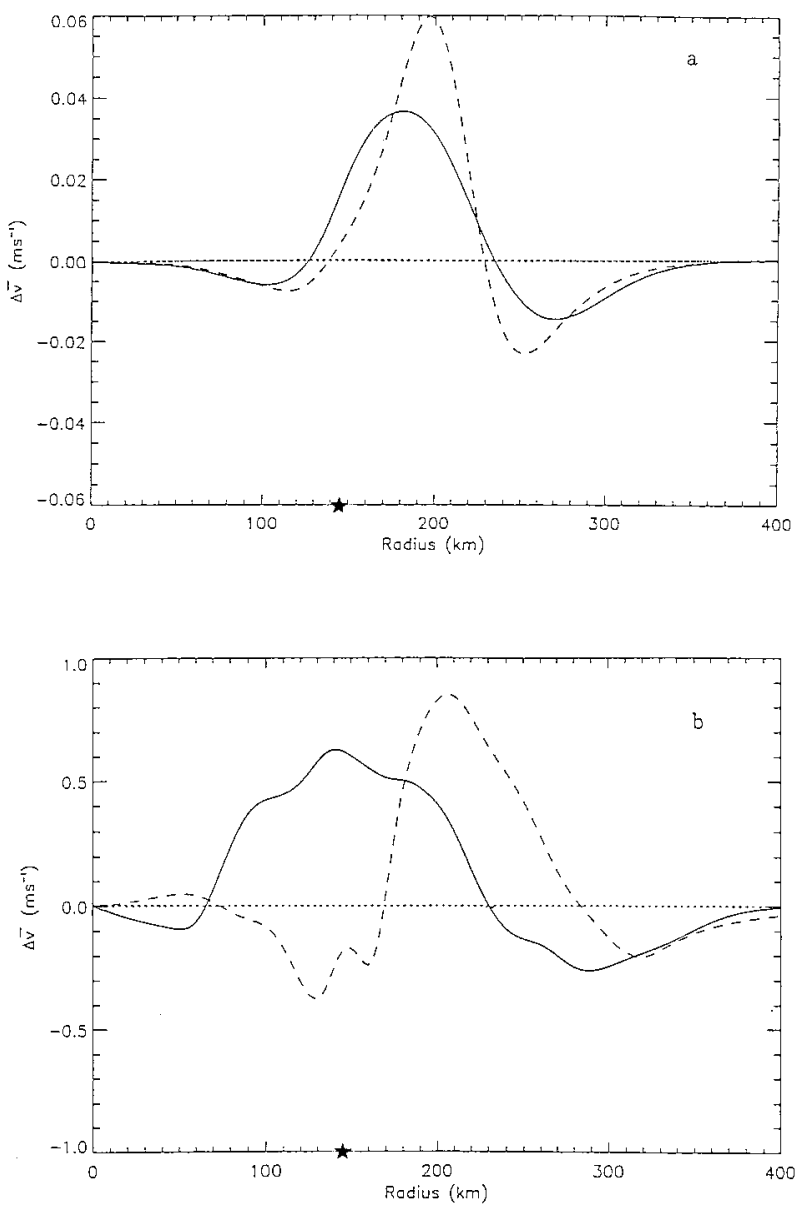

FIG. 10. Change of basic-state tangential velocity $\left(\mathrm{m} \mathrm{s}^{-1}\right)$ for the baroclinic tropical storm vortex due to double-cluster PV anomaly after $24 \mathrm{~h}$ (a) for initial amplitude $10 \%$ of $\bar{q}$ at the RMW for the top (dashed line) and bottom (solid line) of the domain, (b) for the initial amplitude $60 \%$ of $\bar{q}$ at the RMW for the top (dashed line) and bottom (solid line) of the domain.

nature of this circulation, a simple monochromatic wavenumber-2 PV initialization will be presented first. A monochromatic wavenumber-2 anomaly is superimposed on an initially barotropic vortex as shown in Fig. $11 \mathrm{a}$ at upper levels and rotated by $90^{\circ}$ relative to the lower one (Fig. 11b); with an amplitude $20 \%$ of the PV at the RMW, the induced mean secondary circulation at $6 \mathrm{~h}$ is shown schematically in Fig. 11c. By this time the anomaly is axisymmetrized and the change of the basic state has reached its maximum. For this simple case the changes in the mean primary circulation are the strongest $\left(\Delta \bar{v} \sim 0.1 \mathrm{~m} \mathrm{~s}^{-1}\right.$, Fig. 11d) at upper (level 4 and 5) and lower levels (level 0 and 1), and are weakest in the middle. At upper and lower levels the mean secondary circulation counteracts the acceleration in the primary one by mean radial outflow that tends to decelerate the mean primary circulation $(\Delta \bar{u} \sim 0.002 \mathrm{~m}$ $\mathrm{s}^{-1}$ ) inside the the radius of $100 \mathrm{~km}$, and by mean radial inflow that tends to acccelerate the mean primary circulation outside the radius of $100 \mathrm{~km}$. In the middle of the vortex the mean radial flow is reversed due to continuity. The mean vertical velocity is strongest at the upper and lower levels, and is antisymmetric about the middle $\left(\Delta \bar{w} \sim 0.01 \mathrm{~cm} \mathrm{~s}^{-1}\right)$.

The structure of the mean secondary circulation for superimposed double-cluster PV anomalies will be presented next for the experiments with the initially barotropic and baroclinic vortices with the stronger PV anomaly initialization (60\%). The results are shown $6 \mathrm{~h}$ after imposing the PV anomalies, when the circulation reaches its strongest amplitude. In these experiments the upper-level anomaly is not simply a rotation of the lower one as described in the previous paragraph. The PV anomaly is positive at lower levels and negative at upper levels.

For the initially barotropic vortex, the acceleration of the mean primary circulation at lower levels occurs closer to the center of the vortex than that at upper levels. Therefore the mean secondary circulation (Fig. 12a) is spinning down (radial outflow $\Delta \bar{u} \sim 0.014 \mathrm{~m} \mathrm{~s}^{-1}$ ) the primary one inside the RMW at lower levels, where the mean primary circulation accelerates $\left(\Delta \bar{v} \sim 0.24 \mathrm{~m} \mathrm{~s}^{-1}\right)$, and at upper levels the radial inflow $(\Delta \bar{u} \sim-0.008 \mathrm{~m}$ $\left.\mathrm{s}^{-1}\right)$ is spinning up the circulation inside the RMW where the mean primary circulation decelerates $(\Delta \bar{v} \sim$ $\left.-0.20 \mathrm{~m} \mathrm{~s}^{-1}\right)$. The vertical velocity inside the RMW is downward $\left(\Delta \bar{w} \sim-0.03 \mathrm{~cm} \mathrm{~s}^{-1}\right)$ at all levels and outside the RMW is upward $\left(\Delta \bar{w} \sim 0.015 \mathrm{~cm} \mathrm{~s}^{-1}\right)$. The ascending motion of the vortex is tilted inward, which is unlike that of a real tropical storm. Outside the RMW the radial flow at upper and lower levels also counteracts the changes in the mean primary circulation.

For the baroclinic vortex, the change of the basicstate vortex and mean secondary circulation (Fig. 12b) are similar to those for the initially barotropic vortex. In this case at lower levels the radial outflow, $\Delta \bar{u} \sim$ $0.03 \mathrm{~m} \mathrm{~s}^{-1}$, opposes the corresponding acceleration of $\Delta \bar{v} \sim 0.4 \mathrm{~m} \mathrm{~s}^{-1}$; the radial inflow at upper levels, $\Delta \bar{u}$ $\sim-0.04 \mathrm{~m} \mathrm{~s}^{-1}$, opposes the corresponding deceleration of $\Delta \bar{v} \sim-0.17 \mathrm{~m} \mathrm{~s}^{-1}$. Again the vertical velocity inside the RMW is downward $\left(\Delta \bar{w} \sim-0.02 \mathrm{~cm} \mathrm{~s}^{-1}\right)$ at all levels and outside the RMW is upward $(\Delta \bar{w} \sim 0.015$ $\mathrm{cm} \mathrm{s}^{-1}$ ), but the rising and sinking motion now has an outward tilt at lower levels, which seems to be more like that of a real tropical storm.

The asymmetric vertical velocity, $w^{\prime}$, which is strongest initially and then decays, is much stronger than the mean secondary circulation. For the initially barotropic vortex at lower levels, the maximum $w^{\prime} \sim 1.4 \mathrm{~cm} \mathrm{~s}^{-1}$ initially and $w^{\prime} \sim 0.6 \mathrm{~cm} \mathrm{~s}^{-1}$ after $6 \mathrm{~h}$ (Fig. 12c); for the initially baroclinic vortex at lower levels, the maximum $w^{\prime} \sim 2.5 \mathrm{~cm} \mathrm{~s}^{-1}$ initially and $w^{\prime} \sim 1.0 \mathrm{~cm} \mathrm{~s}^{-1}$ after $6 \mathrm{~h}$ (Fig. 12d). This vertical motion could, in principle, feed back to convection, providing a self-sustaining intensification of a storm without further external forcing. The coupling of these waves to the boundary layer via Ekman pumping is an important topic left for future work. 

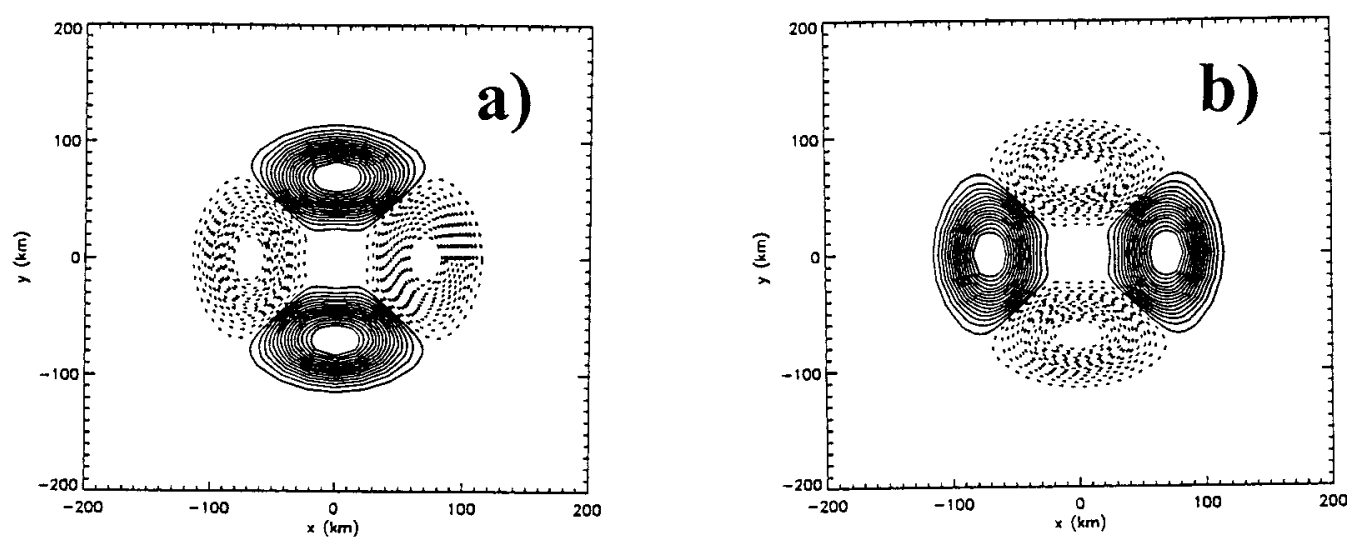

c)

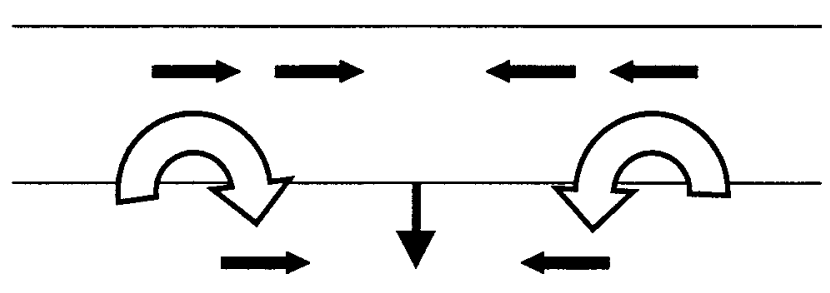

$$
\mathrm{Z}=10 \mathrm{~km}
$$
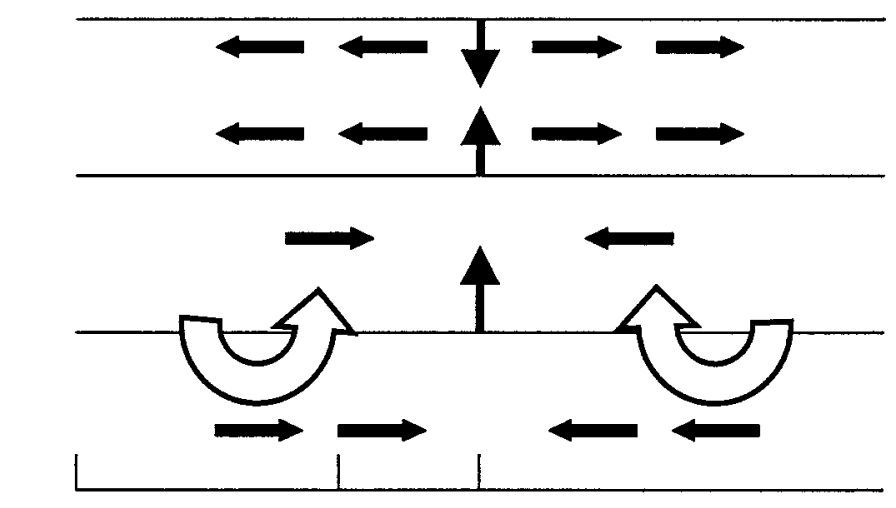

$$
\mathrm{R}=\mathbf{0} \mathrm{km} \quad \mathrm{RMW} \quad \mathbf{1 0 0} \mathrm{km}
$$
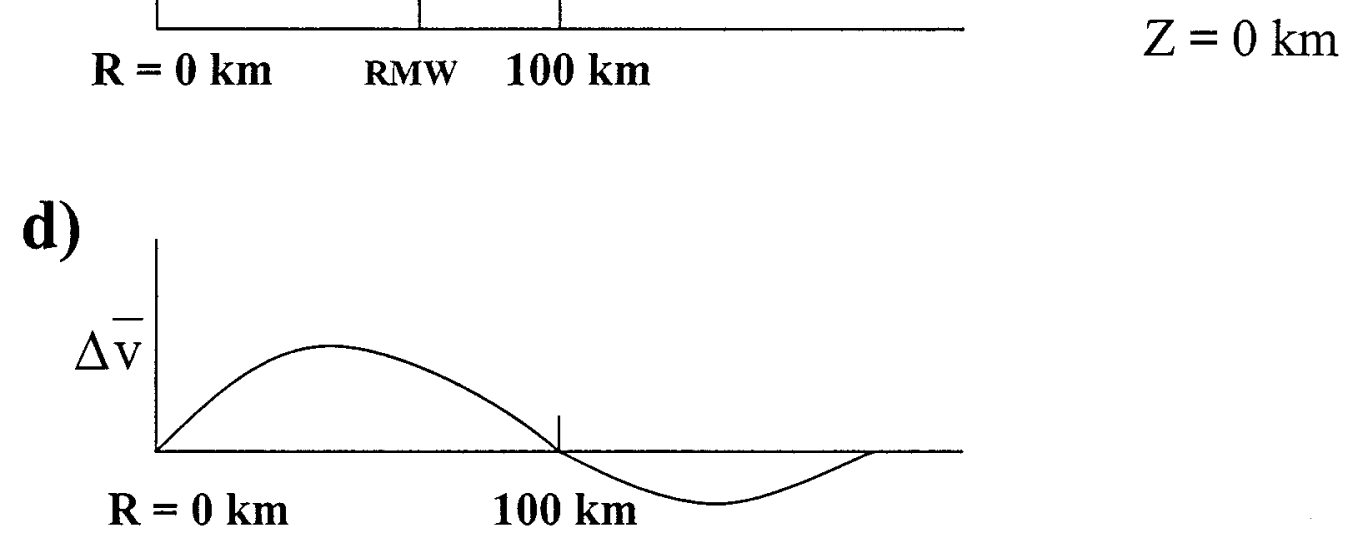

FIG. 11. Initialization of a monochromatic wavenumber-2 PV anomaly at the (a) top of the domain for the initially barotropic tropical storm vortex, rotated by $90^{\circ}$ relative to the (b) bottom of the domain. (c) Secondary (radial and vertical) circulation shown schematically by solid horizontal and vertical arrows and open arrows indicate orientation of circulation gyres after $6 \mathrm{~h}$. (d) Change in the tangential velocity at top (or, equivalently bottom) of the domain. 


\section{c. A-gradient winds}

It should be noted that associated with the mean secondary circulation during the symmetrization process there is also an a-gradient balanced component to the azimuthally averaged tangential wind, as defined below. Previous work disagrees as to whether there is a gradient imbalance in the eyewall (Gray 1991) or whether gradient balance is a good approximation to the azimuthally averaged tangential velocity above the boundary layer (Willoughby 1991). In the context of the AB theory the equation for the perturbation tangential velocity defines the balanced component of the a-gradient wind. The azimuthal average of Eq. (3.7) of SM including the nonlinear terms is

$$
\overline{v^{\prime}}=\frac{1}{\bar{\xi}} \frac{\overline{\partial \phi^{\prime}}}{\partial r}+\overline{\mathcal{N}_{v}^{\prime}},
$$

where the material derivative of $w^{\prime}$ has been neglected, consistent with the findings of Möller and Jones (1998) and their Eq. (4.2). The nonlinear term $\mathcal{N}_{v}^{\prime}=$ $-(1 / \bar{\xi}) \mathcal{N}_{r}^{\prime}$ (see appendix), where $\mathcal{N}_{r}^{\prime}=-u^{\prime} \partial u^{\prime} / \partial r-$ $\left(v^{\prime} / r\right) \partial u^{\prime} / \partial \lambda-w^{\prime} \partial u^{\prime} / \partial z+v^{\prime 2} / r$, is evaluated as discussed in section 2 . If $\overline{\mathcal{N}_{v}^{\prime}}>0$ the balanced symmetric tangential wind will be supergradient; if $\overline{\mathcal{N}_{v}^{\prime}}<0$ it will be subgradient. The balanced symmetric component of the a-gradient wind is

$$
\overline{v_{a}^{\prime}}=\overline{v^{\prime}}-\frac{1}{\bar{\xi}} \frac{\overline{\partial \phi^{\prime}}}{\partial r}=\overline{\mathcal{N}_{v}^{\prime}} .
$$

As an example Fig. 13 shows the change of the tangential wind $\Delta \bar{v}$ and the corresponding balanced component of the a-gradient wind $\overline{v_{a}^{\prime}}$ after $6 \mathrm{~h}$ for the top and bottom of the domain for the experiment with the initially barotropic vortex and the superimposed doublecluster PV anomaly ( $60 \%$ of the PV at the RMW). In a general way the a-gradient wind component opposes the acceleration and deceleration of the basic-state vortex, except outside $r=100 \mathrm{~km}$ at the bottom.

\section{Tropical cyclone development via convective pulsing of PV anomalies}

In order to obtain a better understanding of the threedimensional vortex dynamics at large Rossby number, we follow ME and simulate the ongoing process of convection by adding PV anomalies to the PV fields (pulsing). Our basic-state vortex is the barotropic tropical storm as in the previous section. Again a double-cluster PV anomaly is superimposed on the basic-state vortex, where the upper-level PV anomaly is negative and the lower-level one is positive. In general, the nature of balanced models is such that it is only possible to find a solution to the balance system if the total PV stays positive (e.g., Davis 1992). In order to fulfill this basic rule of balance systems we have to decrease the negative PV anomaly at upper levels relative to the lower one. Otherwise, the total PV at upper levels typically be- comes negative before the lower-level PV anomalies spin up the vortex sufficiently. We are aware that now there is a net PV source, formally violating the integral conservation principle of PV (Hoskins et al. 1985; Haynes and McIntyre 1987, 1990). An alternative method would be to "clip" the PV, filling in small positive values wherever the total PV becomes negative (Davis 1992), but this also formally violates the conservation principle. Either method is a compromise dictated by the constraints of keeping the balance model well posed with an elliptic inversion operator.

For the basic experiment, pulses are imposed every hour, which is less than the tropical storm's 8-h eddy turnover time, but comparable to the storm's local shear time, $(r \partial \bar{\Omega} / \partial r)^{-1}$, which is $\sim 2-3 \mathrm{~h}$ at the initial vortex's RMW. Experiments with much less frequent pulsing, as suggested by ME, needed longer computing time, but were found not to qualitatively change the final results. ${ }^{6}$ The double-cluster PV anomaly is rotated by $90^{\circ}$ every $2 \mathrm{~h}$. Consistent with the discussion in ME's section 5b, the results are essentially unchanged from those when the orientation of the PV anomaly is not altered. Experiments are performed with PV anomalies of different sizes and radial locations. Here the form of the doublecluster PV anomaly is that specified in section 4 , where the radial $e$-folding width ranges between $45 \mathrm{~km}(\beta=$ 40 , "narrow") and $130 \mathrm{~km}(\beta=5$, "broad"), and the location of the PV anomaly maximum ranges from 80$\mathrm{km}$ radius $\left[\left(x_{c}, y_{c}\right)=(0.38,0.38) r_{\max }\right.$; Fig. 14a], which is inside the RMW of $145 \mathrm{~km}$, to $180-\mathrm{km}$ radius $\left[\left(x_{c}, y_{c}\right)\right.$ $=(0.88,0.88) r_{\text {max }}$; not shown]. Figure $14 \mathrm{~b}$ shows a radius-height distribution of the initially narrow PV inside the RMW. The size of the narrow PV anomaly is in the mesoscale regime, even for a hurricane (cf. section 3 of Ooyama 1982). Depending on the location and shape of the anomaly, the hourly increase of the maximum tangential wind $\left(\bar{v}_{\text {max }}\right)$ varies between 0.6 and $1.5 \mathrm{~m} \mathrm{~s}^{-1}$.

The relative strength of the upper and lower PV anomalies are restricted by the considerations given in the first paragraph of this section. The weaker the negative anomaly at upper levels the more the storm intensifies before the total PV becomes negative. In the results presented here the amplitude of the PV asymmetry at lower levels is $150 \%$ of the initial basic state $\mathrm{PV}$ at the RMW ( $\sim 60 \%$ of the maximum PV), and $23 \%$ at upper levels. The low-level PV asymmetry is found to correspond to a temperature anomaly $\sim 15 \mathrm{Kh}^{-1}$ (see section 2 of $\mathrm{ME}$ ), which is consistent with the range of heating rates for mesoscale convective systems found by Mapes and Houze (1995). So as not to complicate the issues related to negative PV mentioned above, the

\footnotetext{
${ }^{6}$ The experiment with the narrow PV anomaly inside the RMW described in the next section was pulsed every hour, every $2 \mathrm{~h}$, and every $4 \mathrm{~h}$ with essentially the same results for the tangential velocity after the same number of pulses.
} 
amplitude of the anomaly is kept constant and is not adjusted to the evolving basic-state PV.

The intensification of the tropical storm is, as expected, weaker at upper levels than at lower levels. For the narrow PV anomaly the intensification is stronger when the PV anomaly is placed inside the RMW. When the PV anomaly is placed inside the RMW, after $48 \mathrm{~h}$ the vortex intensifies to $\bar{v}_{\max }=37.9 \mathrm{~m} \mathrm{~s}^{-1}$ at the bottom of the domain, and contracts, with the RMW moving radially inward from 145 to $105 \mathrm{~km}$ (Fig. 15). This radial contraction is primarily due to inward cyclonic vorticity fluxes associated with vortex Rossby waves (see MK's section $2 \mathrm{~h}$, as well as ME and MM for confirmation). Hoskins et al. (1985) illustrated, in their Fig. 15, how a positive PV anomaly induces a cyclonic circulation that is associated with a warm core aloft due to the lowering of the isentropes. These features can be also seen in Figs. 16a and 16b where the tangential wind, $\bar{v}$, and the change of the basic-state potential temperature, $\Delta \bar{\theta}$, after $48 \mathrm{~h}$ for the narrow PV anomaly inside the RMW are shown. The strengthening of a warm core with a temperature increase of $6.6^{\circ} \mathrm{C}$ is seen. In Fig. 16c the radial-height distribution of the basic-state $\mathrm{PV}, \bar{q}$, and Fig. 16d the basic-state PV, $\bar{q}$, at the bottom of the domain initially and after $48 \mathrm{~h}$ are shown. The development of the basic-state tangential velocity and PV in Figs. 16a and 16c are similar to those in the balanced formulation of Schubert and Alworth (1987) and Möller and Smith (1994). The resulting PV distribution has a local maximum near the RMW and is thus potentially unstable (Montgomery and Shapiro 1995). It would be possible to increase the intensification by decreasing the relative amplitude of the negative upper-level PV anomaly. In an experiment where we pulsed the storm only with a positive PV anomaly at lower levels (not shown) $\bar{v}_{\max } \geq 60 \mathrm{~m} \mathrm{~s}^{-1}$ already after $36 \mathrm{~h}$. When the narrow PV anomaly was at the RMW, the intensification reached only $\bar{v}_{\max }=32.4 \mathrm{~m} \mathrm{~s}^{-1}$ at the bottom of the domain after $48 \mathrm{~h}$. In this case the vortex broadened and the RMW moved outward to $185 \mathrm{~km}$ radius (Fig. 15). This is consistent with the findings of ME regarding the dependence of the spin up on the radial location of the initial asymmetry. In the genesis case, as well as for an intensifying tropical storm, convection typically does not occur at the center of the vortex, confirming the importance of asymmetric processes for the spinup of a vortex.

With the broad PV anomaly the intensification is stronger than with the narrow PV anomaly, whether the anomaly is placed inside or outside the RMW (not shown). The reason for this is simply that for the broader anomaly more total cyclonic PV is added to the lower troposphere. In order to obtain an hourly increase of 1.2 $\mathrm{m} \mathrm{s}^{-1}$, the amplitude is a third of the amplitude of the narrow PV anomaly at lower levels (50\% of the maximum PV at the RMW). When the broad anomaly is located initially at a radius of $80 \mathrm{~km}$ the hurricane-like a)

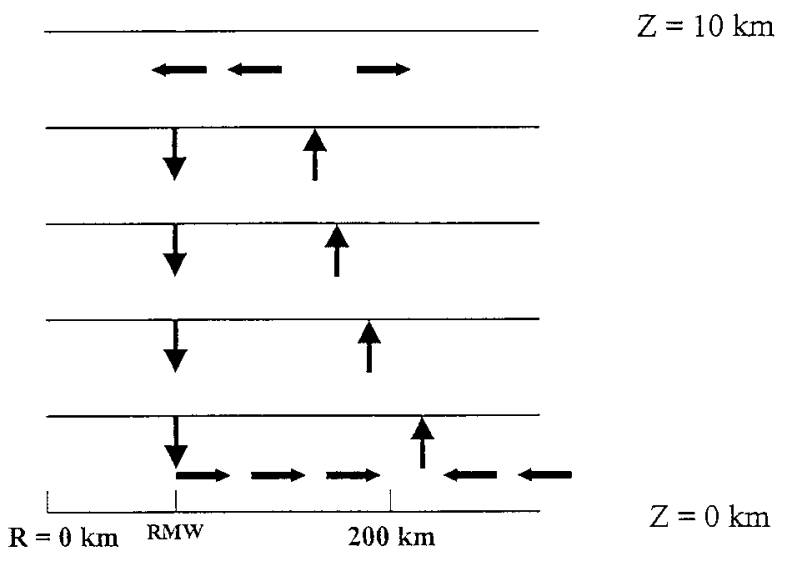

b)

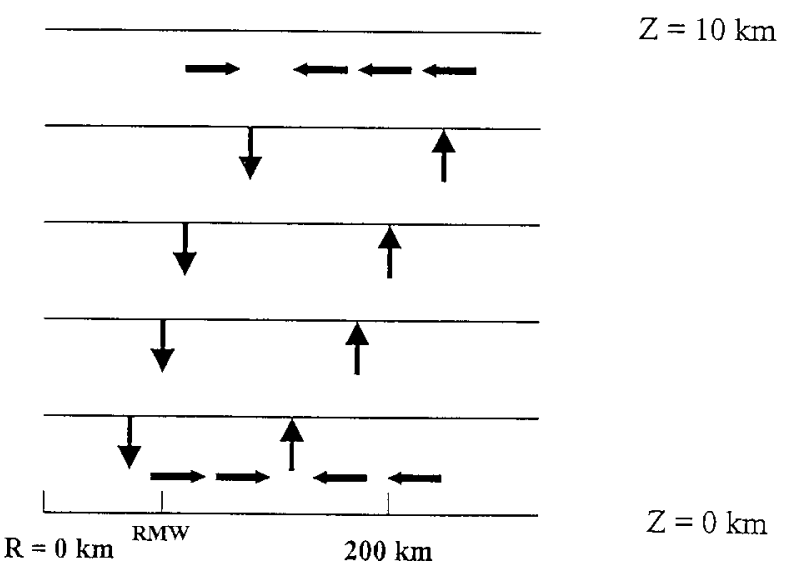

FIG. 12. Secondary (radial and vertical) circulation for the tropical storm vortex shown schematically by arrows after $6 \mathrm{~h}$ for the doublecluster experiment with initial amplitude equal to $60 \%$ of $\bar{q}$ at the

vortex reaches $\bar{v}_{\text {max }}=45.7 \mathrm{~m} \mathrm{~s}^{-1}$ after $48 \mathrm{~h}$. Table 1 summarizes the results of the pulsing experiments.

\section{Conclusions}

In this study we have examined vortex intensification and structure change via wave-mean flow and wavewave interaction of vortex Rossby waves during the axisymmetrization process of asymmetric potential vorticity (PV) disturbances on an initially circular vortex in gradient balance. For finite amplitude relaxation and 

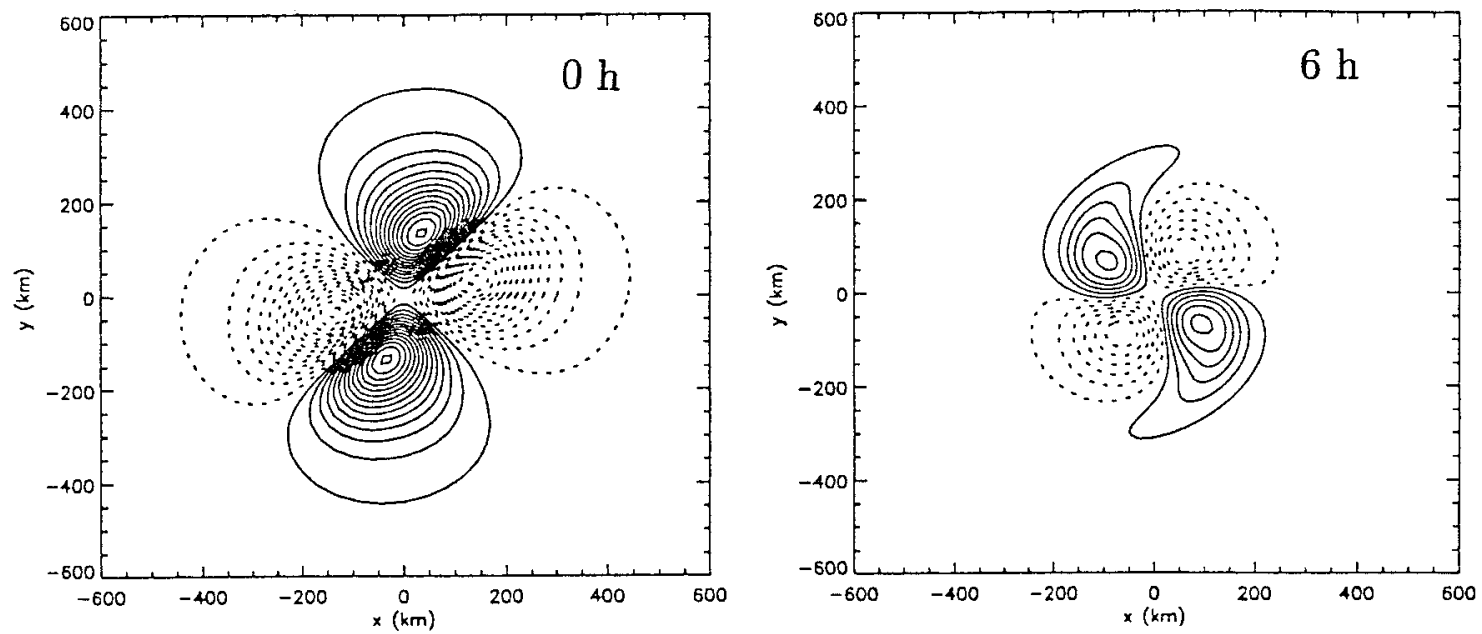

$\mathrm{C}$
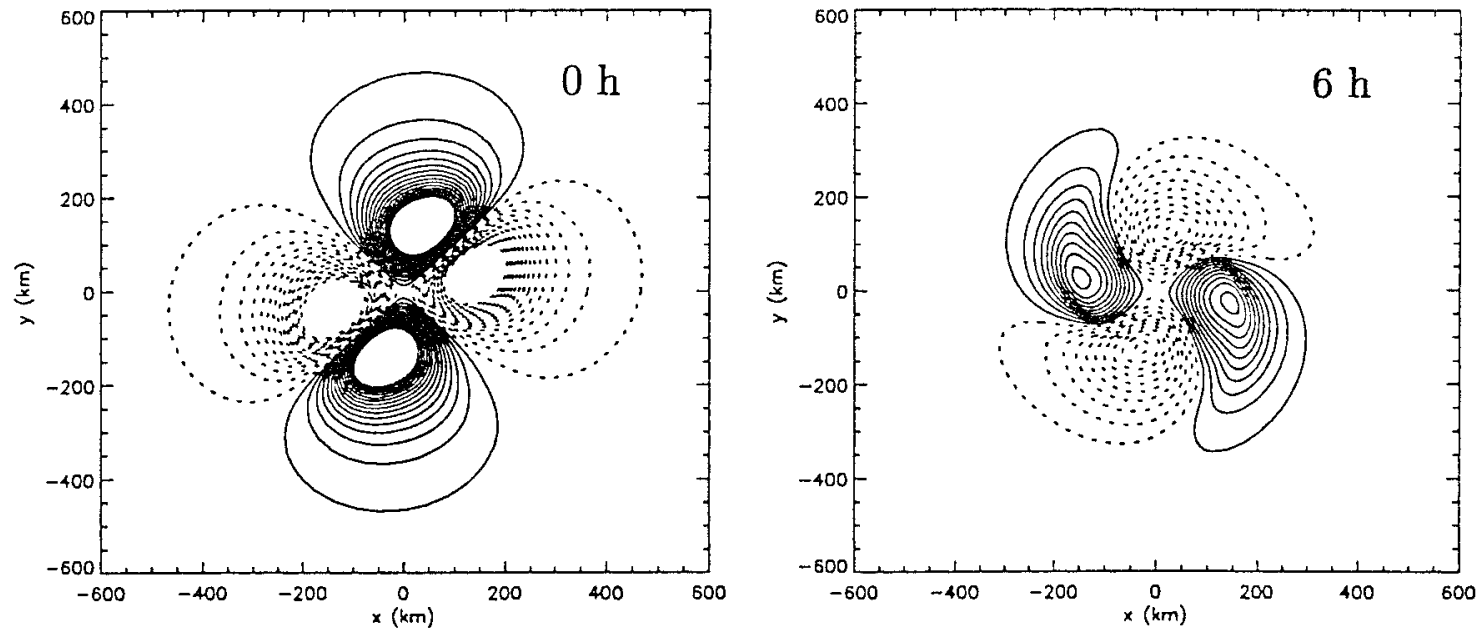

FIG. 12. (Continued) RMW (a) for the initially barotropic vortex, (b) for the initially baroclinic vortex; horizontal cross section of asymmetric vertical circulation initially and after $6 \mathrm{~h}$ (c) for the initially barotropic vortex (d) for the initially baroclinic vortex, contour interval is $1 \times 10^{-3} \mathrm{~m} \mathrm{~s}^{-1}$.

pulsing experiments analogous to those presented, ${ }^{7}$ similar results are obtained when all wave-wave interactions are omitted (not shown). Thus, although the physics of vortex axisymmetrization and vortex merger is generally nonlinear, linear wave theory and wave-mean flow interaction capture a surprisingly substantial part

${ }^{7}$ For the initial barotropic vortex and a superimposed double-cluster PV anomaly of $60 \%$ of the initial basic state PV at the RMW; and for the pulsing experiment with the narrow PV anomaly inside the RMW (see Table 1). of the dynamics for asymmetric PV disturbances near or within the parent vortex's RMW. Part of the reason for the success of the wave-mean flow approximation in capturing the bulk of the mean flow acceleration despite the relatively large amplitude perturbations used can be traced to the strong radial PV gradient of the basic-state circular vortex. Near or within the RMW of the storm the radial PV gradient tends to suppress the nonlinear advective terms. If we use the nondivergent vorticity equation model as a prototype, then the ratio of the nonlinear terms to the linear Rossby restoring terms scale as a beta Rossby number, 

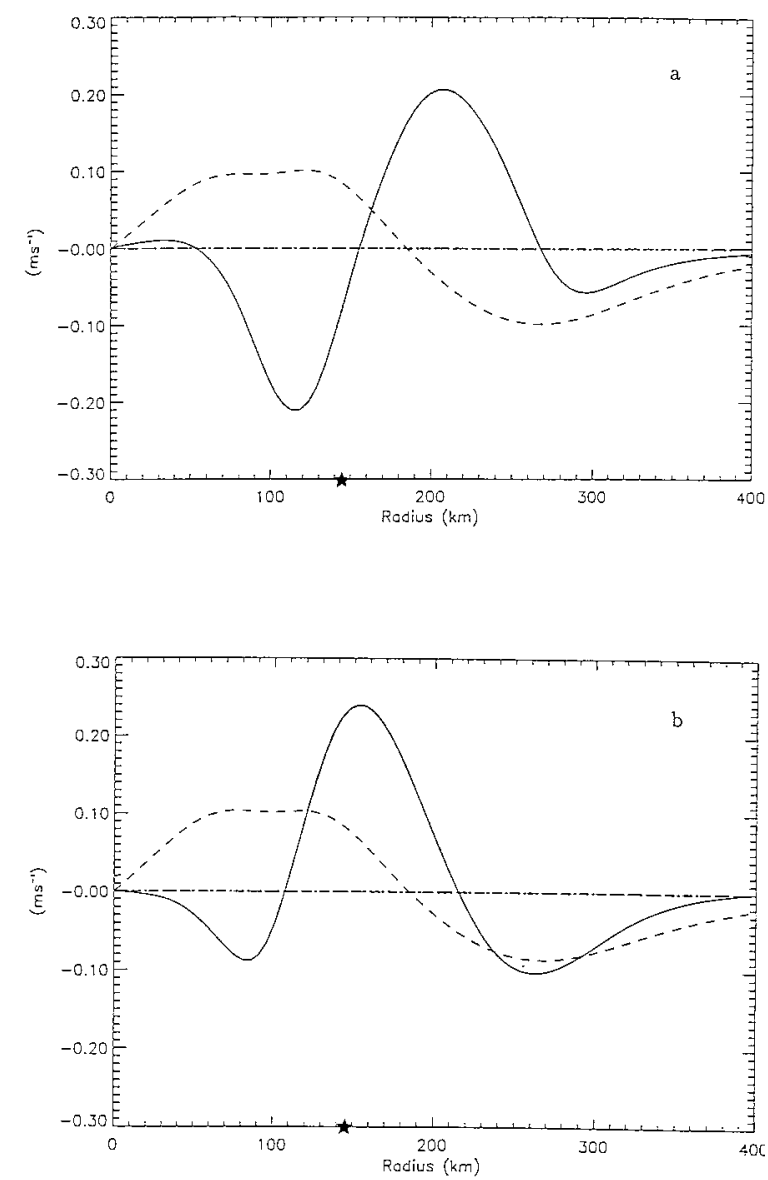

FIG. 13. Change of basic-state tangential velocity $\Delta \bar{v}$ (solid line) and corresponding a-gradient balanced component $\overline{v_{a}^{\prime}}$ (dashed line) $\left(\mathrm{m} \mathrm{s}^{-1}\right)$ after $6 \mathrm{~h}$ for the same case as in Fig. 12a, at the (a) top and (b) bottom of the domain.

$$
R_{\beta} \sim \frac{v_{\mathrm{rms}}^{\prime}}{L^{2}(d \bar{\zeta} / d r)},
$$

where $v_{\text {rms }}$ is a characteristic root-mean-square (rms) eddy velocity amplitude for eddy scale $L$. When $R_{\beta} \gg$ 1 the dynamics are dominated by nonlinear (wave-wave and wave-mean flow) processes, whereas if $R_{\beta} \ll 1$ the dynamics is dominated by purely linear vortex Rossby wave processes. The horizontal scale of the eddies is also important and in general one expects convection to excite a spectrum of eddy scales and corresponding eddy amplitudes. For the mesoscale PV disturbances considered here, however, $R_{\beta}$ is found to be of order unity or less, so the intermediate wave-mean flow approximation is expected and verified to capture the bulk of the wave-vortex interaction.

We have found that the azimuthal scale of the initial superimposed PV anomalies affects the strength of the vertical coupling. Simple relaxation experiments with monochromatic azimuthal wavenumber disturbances (section 3) show the vertical propagation of vortex Ross-
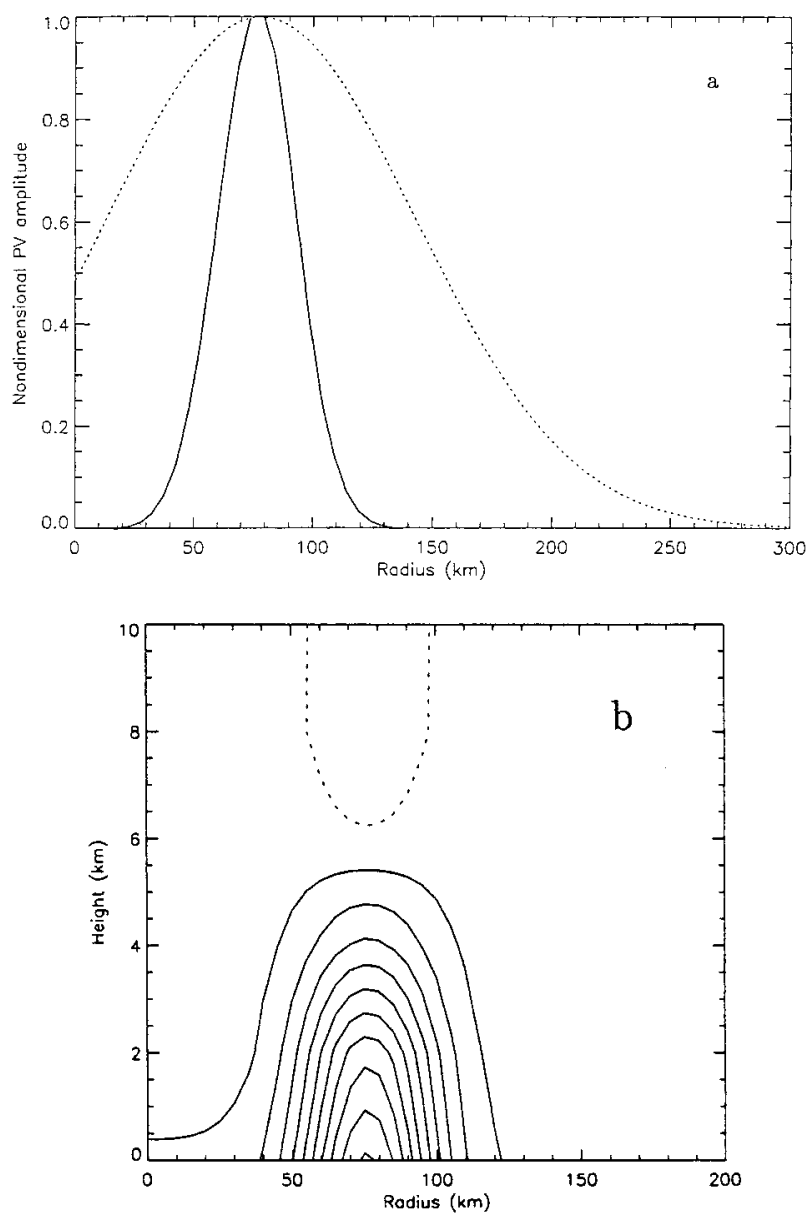

FIG. 14. For the initially barotropic tropical storm vortex (a) amplitude of the initially narrow (solid line) and broad (dotted line) double-cluster PV anomaly $\left(\times 10^{-8} \mathrm{~s}^{-3}\right)$ inside the RMW; (b) radiusheight distribution of the initially narrow PV asymmetry inside the RMW, with contour interval $5 \times 10^{-10} \mathrm{~s}^{-3}$.

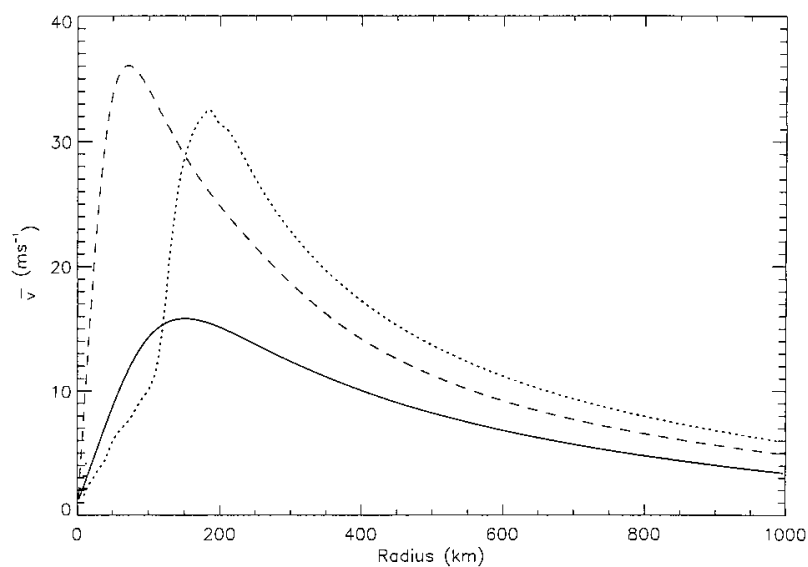

FIG. 15. Basic-state tangential velocity $\left(\mathrm{m} \mathrm{s}^{-1}\right)$ at the bottom of the domain for the narrow PV anomaly initialization for the tropical storm vortex initially (solid line), initialization inside the RMW after $48 \mathrm{~h}$ (dashed line), and initialization at the RMW after $48 \mathrm{~h}$ (dotted line). 

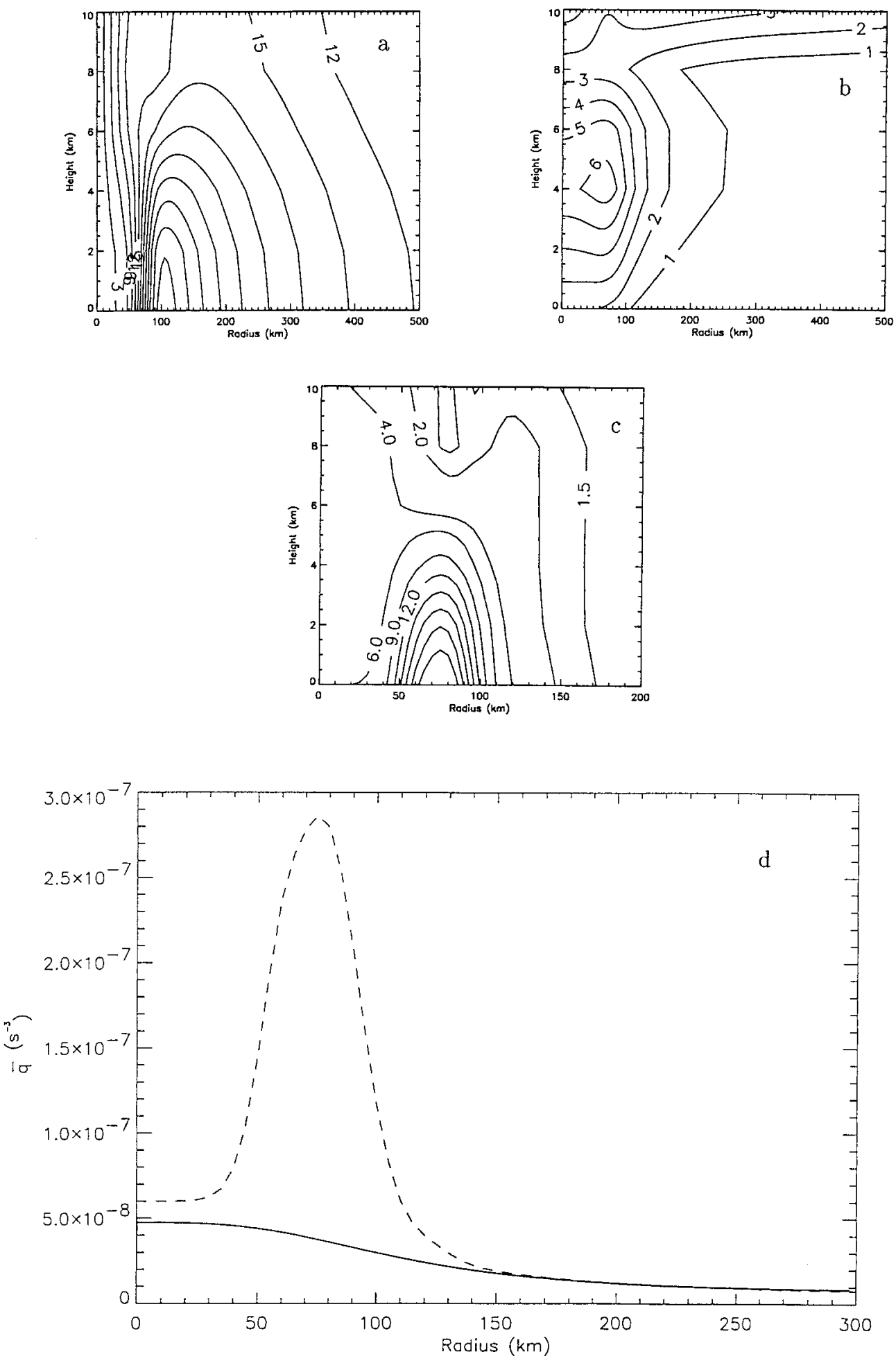

FIG. 16. For the PV initialization inside the RMW (as in dashed line of Fig. 15) radial-height distribution after $48 \mathrm{~h}$ of the (a) tangential velocity, $\bar{v}\left(\mathrm{~m} \mathrm{~s}^{-1}\right)$; (b) of change of the basic-state potential temperature, $\Delta \bar{\theta}$ $\left({ }^{\circ} \mathrm{C}\right)$; (c) PV, $\bar{q}\left(\times 10^{-8} \mathrm{~s}^{-3}\right)$; (d) for the same experiment the radial distribution of the basic-state PV amplitude, $\bar{q}\left(\mathrm{~s}^{-3}\right)$ at the bottom of the domain initially (solid line) and after $48 \mathrm{~h}$ (dashed line). 
TABLE 1. Summary of pulsing experiments varying the spatial extent, position, and amplitude of the double-cluster PV anomaly. The amplitude is given as the \% of the initial PV at the RMW. The final radius of maximum winds and the maximum mean tangential wind achieved at the end of the experiment is presented in the last two columns. The experiment designated by an asterisk (*) indicates that all wave-wave interactions are omitted.

\begin{tabular}{llccc}
\hline \hline $\begin{array}{c}\text { Anomaly } \\
\text { width }\end{array}$ & $\begin{array}{c}\text { Radius of } \\
\text { anomaly } \\
\text { center }\end{array}$ & $\begin{array}{c}\text { Amplitude } \\
\text { at bottom/ } \\
\text { top }(\%)\end{array}$ & $\begin{array}{c}\text { Final RMW/ } \\
\text { experiment } \\
\text { duration }\end{array}$ & $\begin{array}{c}\text { Max } \\
\text { tangential } \\
\text { wind }\left(\mathrm{m} \mathrm{s}^{-1}\right)\end{array}$ \\
\hline Narrow & $80 \mathrm{~km}$ & $150 / 23$ & $105 \mathrm{~km} / 48 \mathrm{~h}$ & 37.9 \\
Narrow* & $80 \mathrm{~km}$ & $150 / 23$ & $105 \mathrm{~km} / 48 \mathrm{~h}$ & 37.7 \\
Narrow & Initial RMW & $150 / 23$ & $175 \mathrm{~km} / 48 \mathrm{~h}$ & 32.4 \\
Narrow & $180 \mathrm{~km}$ & $150 / 23$ & $210 \mathrm{~km} / 36 \mathrm{~h}$ & 30.7 \\
Broad & $80 \mathrm{~km}$ & $50 / 7.5$ & $160 \mathrm{~km} / 48 \mathrm{~h}$ & 45.7 \\
Broad & Initial RMW & $50 / 7.5$ & $220 \mathrm{~km} / 42 \mathrm{~h}$ & 45.6 \\
Broad & $180 \mathrm{~km}$ & $100 / 15$ & $265 \mathrm{~km} / 18 \mathrm{~h}$ & 39.2 \\
\hline
\end{tabular}

by waves. The vertical propagation diminishes with increasing azimuthal or radial wavenumber consistent with the WKB scaling formula for the vertical group velocity [Eq. (3.4)].

In our experiments where double-cluster PV anomalies are superimposed (section 4), the lower-level cyclonic PV anomaly intensifies the vortex while symmetrizing for a wide range of anomaly amplitudes. The above discussion concerning beta Rossby numbers being order unity or less is particularly relevant as it suggests that all such disturbances, in a baroclinically stable vortex, will be axisymmetrized by the parent vortex thereby giving up their cyclonic eddy momentum (and vorticity) to the mean vortex. The numerical simulations are consistent with this view. But depending on the strength of the cluster, the upper-level anticyclonic PV anomaly does not symmetrize and is expelled outward (stronger anomaly) due to the reversal of the PV gradient, or is symmetrized (weaker anomaly) similar to the lower-level positive PV anomaly, as in ME.

As noted in the last paragraph of MK, the gravityinertia component of the response will yield a comparatively weaker wave-mean flow interaction than vortex Rossby waves. Since gravity-inertia waves are filtered out of the balanced model we were able to focus on the vortex Rossby waves dynamics as the fundamental process for vortex development and hurricane intensification by asymmetric forcing.

By contrast to our results, Chimonas and Hauser (1997) found that propagating internal gravity waves might weaken a tornado-like vortex by radiating away the vortex's angular momentum. Chimonas and Hauser (1997) suggest that near-neutral static stability throughout the vortex region is an important component for vortex intensification. However, this is not an appropriate parameterization for hurricanes.

We believe the ideas presented here offer new insight into the dynamics of the interaction between a tropical cyclone and nearby asymmetries, including those associated with upper-level troughs, and their effect on hurricane structure and intensity. While vertical shear is generally considered to have a negative effect on the vortex, that is, weakening, what makes a "good" versus a "bad" trough is not yet well established. Molinari et al. (1995, 1998) suggested that trough interactions induce a secondary circulation that can intensify a hurricane. If this secondary circulation is of sufficient vertical extent it will help initiate a convective response. In our experiments we were able to produce a vertical velocity of $\sim 1-2 \mathrm{~cm} \mathrm{~s}^{-1}$ even with weak PV anomalies. These velocities are of sufficient magnitude to create a self-sustaining process, which could be related to the convective cycles associated with convective bursts, lightning, and intensification (Black et al. 1986; Black and Marks 1987; Lyons et al. 1989; Molinari et al. 1999). We have further established that the strength of the convective response, which we model as an upperlevel negative and a lower-level positive PV anomaly, affects not only the strength but also the character of the interaction. In particular, for a strong anomaly the upper-level negative PV anomaly forms a tripole while it is expelled outward, as in the cyclogenesis experiment of ME. The outward moving anomaly induces an acceleration of the symmetric vortex that is radially outward of that at lower levels. For a weak anomaly, by contrast, the missing change of sign in the basic-state radial PV gradient does not support a neutral or weakly unstable mode and the PV anomaly does not form a tripole. The impact of the resulting change in the upperlevel static and inertial stability of the vortex in a subsequent intensification requires further study.

The results of our study also have implications for the process of eyewall replacement cycles in hurricanes (Willoughby et al. 1982). For PV anomalies generated sufficiently outside the vortex's RMW the subsequent wave-mean flow and wave-wave interactions act primarily to intensify the outer tangential winds rather than the eyewall tangential winds. The induced secondary circulation always tries to oppose the change in the primary circulation (section 4b). In this case the forced secondary circulation is such as to cause subsidence over or near the RMW (see Figs. 11 and 12), which thereby tends to weaken the primary eyewall. For the current experiments there is very little mean radial advection, so there is no substantial inward propagation of the outer wind maximum. Nevertheless, with a frictional boundary layer formulation coupled to convection we would expect inward propagation to occur. This process is directly analogous to the symmetric mechanism elucidated by Shapiro and Willoughby (1982), but now with the vortex Rossby waves playing a central role in the developmental process.

When we simulate the ongoing process of convection by adding double-cluster PV anomalies to the PV fields (pulsing, section 5), the tropical storm intensifies depending on the location and extent of the anomaly. Our experiments show that we are able to intensify a tropical storm into a hurricane-like vortex, which generalizes the small Rossby number results of ME to large Rossby 
number. The "U-shaped" ${ }^{8}$ tangential velocity profiles in Fig. 15 in the final state are consistent with the local PV maximum near the RMW, which tends to lead to barotropic (baroclinic) instability. Schubert et al. (1999) show how unstable modes would tend to mix the high $\mathrm{PV}$ into the center of the vortex. The limit of intensification for a given ratio between the strength of the upper-level negative PV anomaly and the positive lowerlevel one depends on scale and location. The closer the $\mathrm{PV}$ anomaly is to the center the more the storm is able to contract and the more realistic are our results. These results confirm that there exists an alternative means of intensification to the symmetric mode (Ooyama 1969): a tropical storm can intensify to hurricane strength by asymmetric processes.

Acknowledgments. This work was supported in part by the U.S. Office of Naval Research Grant N0001493-1-0456 and Colorado State University. The first author would like to thank Dr. Lloyd Shapiro for many helpful discussions during the course of this work.

\section{APPENDIX}

\section{Numerical Details}

The perturbation velocity components are calculated in the nonlinear form as follows.

$$
\begin{aligned}
u^{\prime}= & \frac{1}{\bar{q}}\left[-N^{2} \frac{1}{r} \frac{\partial \phi^{\prime}}{\partial \lambda}-\frac{N^{2}}{\bar{\xi}} \frac{D_{V}\left(\partial \phi^{\prime} / \partial r\right)}{D t}+\frac{\partial \bar{v}}{\partial z} \frac{D_{V}\left(\partial \phi^{\prime} / \partial z\right)}{D t}\right] \\
& +\mathcal{N}_{u}, \\
v^{\prime}= & \frac{1}{\bar{\xi}} \frac{\partial \phi^{\prime}}{\partial r}-\frac{1}{\bar{\xi}} \frac{D_{V}}{D t}\left(\frac{1}{\bar{\eta} r} \frac{\partial \phi^{\prime}}{\partial \lambda}\right)+\mathcal{N}_{v}, \\
w^{\prime}= & \frac{1}{\bar{q}}\left[-\bar{\eta} \frac{D_{V}\left(\partial \phi^{\prime} / \partial z\right)}{D t}+\frac{\partial \bar{v}}{\partial z} \frac{D_{V}\left(\partial \phi^{\prime} / \partial r\right)}{D t}+\bar{\xi} \frac{\partial \bar{v}}{\partial z} \frac{1}{r} \frac{\partial \phi^{\prime}}{\partial \lambda}\right] \\
& +\mathcal{N}_{w},
\end{aligned}
$$

where

$$
\frac{D_{V}}{D t}=\frac{\partial}{\partial t}+\frac{\bar{v}}{r} \frac{\partial}{\partial \lambda}
$$

and the nonlinear terms

$$
\begin{aligned}
& \mathcal{N}_{u}=\frac{N^{2}}{\bar{q}} \mathcal{N}_{\lambda}-\frac{1}{\bar{q}} \frac{\partial \bar{v}}{\partial z} \mathcal{N}_{\theta}, \quad \mathcal{N}_{v}=-\frac{1}{\bar{\xi}} \mathcal{N}_{r}, \\
& \mathcal{N}_{w}=-\frac{\bar{\xi}}{\bar{q}} \frac{\partial \bar{v}}{\partial z} \mathcal{N}_{\lambda}+\frac{\bar{\eta}}{\bar{q}} \mathcal{N}_{\theta},
\end{aligned}
$$

where $\mathcal{N}_{r}, \mathcal{N}_{\lambda}$, and $\mathcal{N}_{\theta}$ are evaluated using the pseudomomenta and the linear version of $w^{\prime}$ as discussed in

\footnotetext{
${ }^{8}$ The profiles are concave upward (and therefore shaped like a U) near the origin.
}

section 2. The geopotential tendency equation including the nonlinear terms is

$$
\begin{aligned}
& \frac{D_{V}}{D t}\left\{\frac{N^{2}}{\bar{q} \bar{\xi}} \frac{1}{r^{2}} \frac{\partial^{2} \phi^{\prime}}{\partial \lambda^{2}}+\frac{1}{r} \frac{\partial}{\partial r}\left[\frac{r N^{2}}{\bar{q} \bar{\xi}} \frac{\partial \phi^{\prime}}{\partial r}\right]+\frac{\partial}{\partial z}\left[\frac{\bar{\eta}}{\bar{q}} \frac{\partial \phi^{\prime}}{\partial z}\right]\right. \\
& \left.-\frac{1}{r} \frac{\partial}{\partial r}\left[\frac{r}{\bar{q}} \frac{\partial \bar{v}}{\partial z} \frac{\partial \phi^{\prime}}{\partial z}\right]-\frac{1}{r} \frac{\partial}{\partial z}\left[\frac{r}{\bar{q}} \frac{\partial \bar{v}}{\partial z} \frac{\partial \phi^{\prime}}{\partial r}\right]\right\} \\
& +\left[\frac{\partial}{\partial r}\left(\frac{N^{2}}{\bar{q}}\right)-\frac{\partial}{\partial z}\left(\frac{\bar{\xi}}{\bar{q}} \frac{\partial \bar{v}}{\partial z}\right)\right] \frac{1}{r} \frac{\partial \phi^{\prime}}{\partial \lambda} \\
& =\nabla_{3 \mathrm{D}} \cdot\left\{\mathcal{N}_{u}, \mathcal{N}_{v}, \mathcal{N}_{w}\right\} \text {, }
\end{aligned}
$$

where the operator on the right-hand side is the threedimensional divergence.

The inversion of the PV for the geopotential is accomplished for each azimuthal wavenumber with a direct LU decomposition (lower triangular, upper triangular) of a band diagonal matrix based on the discretization of the elliptic operator in (3.1) in radial-height space. Second-order centered differences are employed to approximate radial and vertical derivatives. Denoting the wavenumber component by a subscript, and the Fourier transform by a caret, the boundary conditions are then as follows. For wavenumbers $n \geq 1, \hat{\phi}_{n}=0$ at $r$ $=0$, and $r=r_{\max }$ (outer boundary); while for $n=0$, $\partial \hat{\phi}_{0} / \partial r=0$ at $r=0$ and $\hat{\phi}_{0}=0$ at $r=r_{\max }$. For the upper and lower boundaries $\partial \hat{\phi}_{n} / \partial z=0$ (zero potential temperature anomaly) for all $n$.

Similarly, the numerical model used to solve the tendency equation for the geopotential (given above) is semispectral, with Fourier modes in the azimuthal coordinate and grid points in the radial and vertical coordinates. The nonlinear terms are computed by explicitly summing a convolution sum in Fourier space. Inversion for the geopotential tendency $\partial \phi^{\prime} / \partial t$ is accomplished with an LU decomposition of a band diagonal matrix derived from the elliptic operator in Eq. (A.1), where the tendencies in the nonlinear terms on the righthand side are used from the previous time step. The radial boundary conditions are for wavenumbers $n \geq$ $1, \partial \hat{\phi}_{n} / \partial t=0$ at $r=0$, and $r=r_{\max }$ (outer boundary); while for $n=0, \partial^{2} \hat{\phi}_{0} / \partial r \partial t=0$ at $r=0$ and $\partial \hat{\phi}_{0} / \partial t=$ 0 at $r=r_{\max }$. For the upper and lower boundaries $\partial^{2} \hat{\boldsymbol{\phi}}_{n} / \partial z \partial t=0$ (zero potential temperature tendency) for all $n$.

The numerical model is time stepped with a fourthorder Runge-Kutta scheme with typical time increment equal to $2 \mathrm{~min}$. With a radial grid spacing of 2.5 and 5 $\mathrm{km}$, the chosen time step of 5 min falls below the empirically determined CFL stability threshold. The vertical grid spacing is 1 or $2 \mathrm{~km}$, requiring 6 or 11 levels, respectively, for the given model height of $10 \mathrm{~km}$. For the experiments considered here the results are converged at the chosen resolution. The azimuthal wavenumber truncation is $n=8$. Pulsing experiments with up to $n=24$ give essentially the same results. All runs 
use 1200 radial grid points and employ explicit diffusion of $\phi^{\prime}$ so as to remove finescale PV associated with the potential enstrophy cascade. The explicit form of the diffusion term added to the right-hand side of the tendency equation is proportional to $\nabla^{2}\left(\nabla^{2} \phi^{\prime}\right)$, which has the desirable property of ensuring that the contribution to the area-averaged perturbation energy is negative definite. The effective second-order diffusivity is $233 \mathrm{~m}^{2}$ $\mathrm{s}^{-1}$, and the impact of diffusion on local and global PV and global energy conservation is found to be negligible for the duration of all integrations presented. Relaxation experiments with the barotropic vortex where the diffusion was set to zero produce essentially the same results.

\section{REFERENCES}

Black, P. G., and F. D. Marks Jr., 1987: Environmental interactions associated with hurricane supercells. Preprints, 17th Conf. on Hurricanes and Tropical Meteorology, Miami, FL, Amer. Meteor. Soc., 416-419.

—, R. A. Black, J. Hallett, and W. A. Lyons, 1986: Electrical activity of the hurricane. Preprints, 23d Conf. on Radar and Cloud Physics, Snowmass, CO, Amer. Meteor. Soc., J277-J280.

Carr, L. E., III, and R. T. Williams, 1989: Barotropic vortex stability to perturbations from axisymmetry. J. Atmos. Sci., 46, $3177-$ 3191.

Challa, M., and R. L. Pfeffer, 1980: Effects of eddy fluxes of angular momentum on the model hurricane development. J. Atmos. Sci., 37, 1603-1618.

—, and —, Q. Zhao, and S. W. Chang, 1998: Can eddy fluxes serve as a catalyst for hurricane and typhoon formation? J. Atmos. Sci., 55, 2201-2219.

Charney, J. G., and P. G. Drazin, 1961: Propagation of planetary scale disturbances from the lower into the upper atmosphere. J. Geophys. Res., 66, 83-109.

Chimonas, G., and H. M. Hauser, 1997: The transfer of angular momentum from vortices to gravity swirl waves. Mon. Wea. Rev., 54, 1701-1711.

Davis, C. A., 1992: Piecewise potential vorticity inversion. J. Atmos. Sci., 49, 1397-1411.

Gall, R., J. Tuttle, and P. Hildebrand, 1998: Small-scale spiral bands observed in Hurricanes Andrew, Hugo, and Erwin. Mon. Wea. Rev., 126, 1749-1766.

Gray, W. M., 1968: Global view of the origin of tropical disturbances and storms. Mon. Wea. Rev., 96, 669-700.

_ 1991: Comments on "Gradient balance in tropical cyclones." J. Atmos. Sci., 48, 1201-1208.

Guinn, T. A., and W. H. Schubert, 1993: Hurricane spiral bands. J. Atmos. Sci., 50, 3380-3403.

Hakim, G. J., D. Keyser, and L. F. Bosart, 1996: The Ohio Valley wave-merger cyclogenesis event of 25-26 January 1978. Part II: Diagnosis using quasigeostrophic potential vorticity inversion. Mon. Wea. Rev., 124, 2176-2205.

Hawkins, H. F, and D. T. Rubsam, 1968: Hurricane Hilda, 1964. Part II: The structure and budgets of the hurricane on October 1 , 1964. Mon. Wea. Rev., 96, 617-636.

Haynes, P. H., and M. E. McIntyre, 1987: On the evolution of vorticity and potential vorticity in the presence of diabatic heating and frictional or other forces. J. Atmos. Sci., 44, 828-841.

$\longrightarrow$, and - 1990: On the conservation and impermeability theorems for potential vorticity. J. Atmos. Sci., 47, 2021-2031.

Holland, G. J., and G. S. Dietachmayer, 1993: On the interaction of tropical-cyclone-scale vortices. III: Continuous barotropic vortices. Quart. J. Roy. Meteor. Soc., 119, 1381-1398.

Hoskins, B. J., and F. P. Bretherton, 1972: Atmospheric frontogenesis models: Mathematical formulation and solution. J. Atmos. Sci., 29, 11-37.

- M. E. McIntyre, and A. W. Robertson, 1985: On the use and significance of isentropic potential vorticity maps. Quart. J. Roy. Meteor. Soc., 111, 877-946.

Lyons, W. A., M. G. Venne, P. G. Black, and R. C. Gentry, 1989: Hurricane lightning: A new diagnostic tool for tropical storm forecasting? Preprints, 18th Conf. on Hurricanes and Tropical Meteorology, San Diego, CA, Amer. Meteor. Soc., 113-114.

Mapes, B. E., and R. A. Houze Jr., 1995: Diabatic divergence profiles in Western Pacific mesoscale convective systems. J. Atmos. Sci., 52, 1807-1828.

McCalpin, J. D., 1987: On the adjustment of azimuthally perturbed vortices. J. Geophys. Res., 92, 8213-8225.

Melander, M. V., J. C. McWilliams, and N. J. Zabusky, 1987: Axisymmetrization and vorticity-gradient intensification of an isolated two-dimensional vortex through filamentation. J. Fluid Mech., 178, 137-159.

Molinari, J., S. Skubis, and D. Vollaro, 1995: External influences on hurricane intensity. Part III: Potential vorticity evolution. J. Atmos. Sci., 52, 3593-3606.

,,--- F. Alsheimer, and H. E. Willoughby, 1998: Potential vorticity analysis of tropical cyclone intensification. J. Atmos. Sci., 55, 2632-2644.

- P. Moore, and V. Idone, 1999: Convective structure of hurricanes as revealed by lightning locations. Mon. Wea. Rev., 127, $520-534$.

Möller, J. D., and R. K. Smith, 1994: The development of potential vorticity in a hurricane-like vortex. Quart. J. Roy. Meteor. Soc., 120, $1255-1265$.

— cyclones using the asymmetric balance theory. J. Atmos. Sci., 55 259-282.

— and M. T. Montgomery, 1999: Vortex Rossby-waves and their influence on hurricane intensification in a barotropic model. $J$. Atmos. Sci., 56, 1674-1687.

Montgomery, M. T., and L. J. Shapiro, 1995: Generalized CharneyStern and Fjortoft theorems for rapidly rotating vortices. J. Atmos. Sci., 52, 1830-1833.

—_ and R. J. Kallenbach, 1997: A theory for vortex Rossby waves and its application to spiral bands and intensity changes in hurricanes. Quart. J. Roy. Meteor. Soc., 123, 435-465.

— Eigenmode structure. J. Atmos. Sci., 54, 1868-1885.

_ forced vortex Rossby waves in a three-dimensional quasigeostrophic model. J. Atmos. Sci., 55, 3176-3207.

Ooyama, K. V., 1969: Numerical simulation of the life cycle of tropical cyclones. J. Atmos. Sci., 26, 3-40.

- 1982: Conceptual evolution of the theory and modeling of the tropical cyclone. J. Meteor. Soc. Japan, 60, 369-379.

Orlandi, P., and G. J. F. van Heijst, 1992: Numerical simulation of tripolar vortices in 2D flow. Fluid Dyn. Res., 9, 179-206.

Palmèn, E., and C. W. Newton, 1969: Atmospheric Circulation Systems. Academic Press, 603 pp.

Pfeffer, R. L., 1958: Concerning the mechanics of hurricanes. J. Meteor., 15, 113-120.

Polvani, L. M., and X. J. Carton, 1990: The tripole: A new coherent vortex structure of incompressible two-dimensional flows. Geophys. Astrophys. Fluid Dyn., 51, 87-102.

Ritchie, E. A., and G. J. Holland, 1993: On the interaction of tropicalcyclone-scale vortices. II: Discrete vortex patches. Quart. J. Roy. Meteor. Soc., 119, 1363-1379.

Rotunno, R., and K. A. Emanuel, 1987: An air-sea interaction theory 
for tropical cyclones. Part II: Evolutionary study using a nonhydrostatic axisymmetric numerical model. J. Atmos. Sci., 44, $542-561$.

Schubert, W. H., and B. T. Alworth, 1987: Evolution of potential vorticity in tropical cyclones. Quart. J. Roy. Meteor. Soc., 113, 147-162.

—, M. T. Montgomery, R. K. Taft, T. A. Guinn, S. R. Fulton, J. P. Kossin, and J. P. Edwards, 1999: Polygonal eyewalls, asymmetric eye contraction and potential vorticity mixing in hurricanes. J. Atmos. Sci., 56, 1197-1223.

Shapiro, L. J., 2000: Potential vorticity asymmetries and tropical cyclone evolution in a moist three-layer model. J. Atmos. Sci., 57, 3645-3662.

, and H. E. Willoughby, 1982: The response of balanced hur- ricanes to local sources of heat and momentum. J. Atmos. Sci., 39, 378-394.

— , and M. T. Montgomery, 1993: A three-dimensional balance theory for rapidly rotating vortices. J. Atmos. Sci., 50, 3322-3335.

Smith, G. B., and M. T. Montgomery, 1995: Vortex axisymmetrization: Dependence on azimuthal wavenumber or asymmetric radial structure changes. Quart. J. Roy. Meteor. Soc., 121, 1615-1650.

Sutyrin, G. G., 1989: Azimuthal waves and symmetrization of an intense vortex. Sov. Phys. Dokl., 34, 104-106.

Willoughby, H. E., 1991: Reply to "Comments on Gradient balance in tropical cyclones." J. Atmos. Sci., 48, 1209-1212.

, J. A. Clos, and M. G. Shoreibah, 1982: Concentric eye walls, secondary wind maxima, and the evolution of the hurricane vortex. J. Atmos. Sci., 39, 395-411. 